\title{
FEUILLETAGES HOLOMORPHES DE CODIMENSION 1: UNE ÉTUDE LOCALE DANS LE CAS DICRITIQUE
}

\author{
D. CERVEAU, A. LINS NETO \& M. RAVARA-VAGO
}

\begin{abstract}
RÉsumÉ. Nous décrivons les singularités de feuilletages holomorphes dicritiques de petite multiplicité en dimension 3. En particulier nous relions l'existence de déformations et de déploiements non triviaux à des problèmes d'intégrabilité liouvillienne.
\end{abstract}

\section{TABLE DES MATIÈRES}

1. Introduction

2. Notations, définitions et rappels

3. Cas dicritique dégénéré avec $\nu \leq 2$

4. Un lemme utile et le Théorème de Camacho-Lins Neto revisité

5. Feuilletages avec singularités nilpotentes; problèmes de déploiements

6. Théorème de Dulac : présentation et applications

7. Points nilpotents génériques

8. Vers la conjecture : le cas $\mu=3$

9. Le cas $\mu=4$

10. Le $\operatorname{cas} \mu=5$

11. Le cas $\mu=6$

12. Krull-déformations pour certains feuilletages transversalement affines

13. Perturbations de 1 -formes fermées : exemples

14. Conclusion

Références

\section{INTRODUCTION}

On se propose dans cet article de donner la description de certains germes de feuilletages holomorphes de codimension un à l'origine de $\mathbb{C}^{n}$. Un tel feuilletage $\mathcal{F}$ est associé à la donné d'une 1 -forme holomorphe $\omega \in \Omega^{1}\left(\mathbb{C}^{n}, 0\right)$, définie à unité multiplicative près, satisfaisant la condition d'intégrabilité de Frobenius $\omega \wedge d \omega=0$ et dont le lieu singulier Sing $\omega=$ Zéros $(\omega)$ est de codimension supérieure ou égale à deux. On note $\mathcal{F}=\mathcal{F}_{\omega}$ et Sing $\mathcal{F}=\operatorname{Sing} \omega$. La condition d'intégrabilité est non linéaire en les coefficients de $\omega$, ce qui rend difficile la construction d'exemples par des procédés calculatoires. Il y a essentiellement deux manières simples pour construire de tels exemples. La première consiste à se donner un germe $\omega_{0} \in \Omega^{1}\left(\mathbb{C}^{2}, 0\right)$ en dimension 2 (dans ce cas la condition d'intégrabilité est triviale), une application holomorphe $F: \mathbb{C}^{n}, 0 \rightarrow \mathbb{C}^{2}, 0$ dominante et à considérer $\omega=F^{*} \omega_{0}$ qui est automatiquement intégrable; les feuilles du feuilletage $\mathcal{F}_{\omega}$ sont alors fibrées par les niveaux de $F$. Un tel feuilletage sera dit de type pull-back; on peut d'ailleurs généraliser cette procédure en considérant un feuilletage $\mathcal{F}_{0}$ d'une surface $S_{0}$, éventuellement singulière, et une application méromorphe $F: \mathbb{C}^{n} \rightarrow S_{0}$ (typiquement la projection naturelle $\mathbb{C}^{3} \rightarrow \mathbb{P}_{\mathbb{C}}^{2}$ ) et en rappelant $\mathcal{F}_{0}$ par $F$. L'autre

Date: Avril 2014.

Key words and phrases. Singular holomorphic foliations, finite determinacy, unfolding, Liouvillian integration. 
manière est de considérer les feuilletages $\mathcal{F}_{\theta}$ associés aux 1-formes méromorphes $\theta$ fermées : $d \theta=0$. Comme nous le rappellerons une telle forme s'écrit :

$$
\theta=\sum \lambda_{i} \frac{d f_{i}}{f_{i}}+d h
$$

où les $f_{i}$ sont holomorphes, $h$ méromorphe et les résidus $\lambda_{i}$ des nombres complexes. Essentiellement les feuilles de $\mathcal{F}_{\theta}$ sont les niveaux de la fonction multivaluée $\sum \lambda_{i} \log f_{i}+h$. Ceci n'est pas sans rappeler la conjecture, concernant cette fois les feuilletages globaux, suivante :

Conjecture. (Brunella, Lins Neto et alt $[10]$ ) Soit $\mathcal{F}$ un feuilletage holomorphe de codimension un sur une variété projective $X$. Alors ou bien $\mathcal{F}$ est transversalement projectif (sur un ouvert de Zariski) ou bien il existe $F: X \rightarrow S$ une application rationnelle vers une surface $S$ et un feuilletage $\mathcal{G}$ de $S$ tels que $\mathcal{F}=F^{*} \mathcal{G}$.

Pour éclairer cette conjecture disons que parmi les feuilletages transversalement projectifs il y a les feuilletages donnés par une 1-forme fermée méromorphe ou les feuilletages transverses à une fibration rationnelle (sur un ouvert de Zariski fibré). Ils sont donnés par une 1 -forme rationnelle $\omega_{0}$ pour laquelle existe deux autres 1 -formes rationnelles $\omega_{1}, \omega_{2}$ formant un $S L(2, \mathbb{C})$ triplet [10, 20] :

$$
d \omega_{0}=\omega_{0} \wedge \omega_{1}, \quad d \omega_{1}=\omega_{0} \wedge \omega_{2}, \quad d \omega_{2}=\omega_{1} \wedge \omega_{2} .
$$

Soit $\omega \in \Omega^{1}\left(\mathbb{C}^{n}, 0\right)$ un germe de 1 -forme intégrable, $\omega=\sum a_{i} d z_{i}$; si l'ensemble singulier Sing $\omega:=\left\{a_{1}=\cdots=a_{n}=0\right\}$ est suffisament petit, Cod Sing $\omega \geq 3$, le Théorème de Frobenius de B. Malgrange assure l'existence d'une intégrale première non constante $f \in \mathcal{O}\left(\mathbb{C}^{n}, 0\right): \omega=g d f$, $g \in \mathcal{O}^{*}\left(\mathbb{C}^{n}, 0\right)$; ici les feuilles sont les niveaux de $f$. On pourrait penser que celà décrit la situation générique (pour la topologie de Krull), mais il n'en est rien : on sait en effet depuis Kupka et Reeb que la condition $d \omega(0) \neq 0$ implique que le lieu singulier est lisse de codimension deux et cette propriété est évidemment stable. Nous précisons dans le texte ce phénomène bien connu et nous présentons la classification des 1 -formes intégrables dont le 1-jet est non trivial suivant les travaux de [17, 12, 19. Une grande partie du travail que nous proposons repose sur l'idée naïve suivante; si l'on procède au développement de Taylor de $\omega$ :

$$
\omega=\omega_{\nu}+\omega_{\nu+1}+\cdots+\omega_{k}+\cdots
$$

où chaque $\omega_{k}$ est une 1 -forme homogène de degré $k$, i.e. à coefficients polynômes homogènes de degré $k$, et $\omega_{\nu}$ est la partie homogène de plus bas degré non nulle, alors la partie initiale $\operatorname{In}(\omega)=\omega_{\nu}$ de $\omega$ est intégrable. Mieux, il y a une homotopie

$$
\omega_{t}=\omega_{\nu}+t \omega_{\nu+1}+\cdots+t^{k} \omega_{\nu+k}+\cdots, \quad t \in \mathbb{C}
$$

reliant $\omega=\omega_{t=1}$ à sa partie initiale $\omega_{t=0}=\operatorname{In}(\omega)$. On peut donc espérer, modulo des conditions raisonnables sur $\omega_{\nu}$, que la 1 -forme $\omega$, que l'on voit donc comme une déformation de $\omega_{\nu}$, va conserver certaines propriétés de sa partie initiale $\operatorname{In}(\omega)$. Pour présenter nos résultats nous avons besoin de quelques notations et rappels. On désigne par $R$ le champ radial, $R=\sum z_{i} \frac{\partial}{\partial z_{i}}$. Une 1 -forme homogène intégrable $\omega_{\nu}$ est dite dicritique si le polynôme $P_{\nu+1}=i_{R} \omega_{\nu}$ est identiquement nul; elle est non dicritique sinon. Une forme dicritique induit un feuilletage sur l'espace projectif $\mathbb{P}_{\mathbb{C}}^{n-1}$, tandis que le feuilletage homogène associé à une forme non dicritique est défini par une 1-forme fermée rationnelle : en effet $\frac{\omega_{\nu}}{P_{\nu+1}}$ est fermée. Modulo des conditions génériques portant sur $\omega_{\nu}$, ces propriétés sont gardées en mémoire par les $\omega$ telles que $\operatorname{In}(\omega)=\omega_{\nu}$. Ainsi, dans le cas non dicritique, si $\left[P_{\nu+1}=0\right] \subset \mathbb{P}_{\mathbb{C}}^{n-1}$ est réduit et à croisement normaux, alors $\mathcal{F}_{\omega}$ est encore défini par une 1 -forme fermée méromorphe dès que les résidus $\lambda_{i}$ de $\omega_{\nu}=\operatorname{In}(\omega)$ satisfont une condition générique (satisfaite sur un ouvert dense); de même si $\left[P_{\nu+1}=0\right]$ est irréductible de degré $p^{s}$, avec $p$ premier et $n \geq 3$ [1] : en fait dans ce cas $\omega$ possède une intégrale première holomorphe non constante.

Dans le cas dicritique et en dimension 3, on démontre dans [1] que si $d \omega_{\nu}=d(\operatorname{In}(\omega))$ est à singularité isolée et $\nu \geq 3$, alors les feuilletages $\mathcal{F}_{\omega_{\nu}}$ et $\mathcal{F}_{\omega}$ sont holomorphiquement conjugués, ce que l'on peut interpréter comme un résultat de détermination finie ou de stabilité (pour la topologie de Krull). 
Nous nous intéressons dans cet article à des feuilletages $\mathcal{F}_{\omega}$ dont la partie homogène $\omega_{\nu}=\operatorname{In}(\omega)$ ne satisfait plus les conditions précédentes. Si $\omega_{\nu}$ est une 1-forme homogène intégrable dicritique, on note $\left[\mathcal{F}_{\omega_{\nu}}\right]$ le feuilletage de $\mathbb{P}_{\mathbb{C}}^{n-1}$ associé; si Cod Sing $\omega_{\nu} \geq 2$, c'est un feuilletage de degré $\nu-1$. Rappelons qu'un point central (ou une singularité de type centre) pour un feuilletage $\mathcal{G}$ du plan est un point singulier $m$ en lequel $\mathcal{G}$ possède une intégrale première de Morse; toujours en dimension 2 , un point singulier $m$ est dit nilpotent si $\mathcal{G}_{, m}$ est défini par un germe de 1 -forme dont la partie initiale est de type $x d x$.

Les résultats qui suivent sont propres à la dimension 3, première dimension ou la condition d'intégrabilité est non triviale.

Théorème A. Soit $\omega \in \Omega^{1}\left(\mathbb{C}^{3}, 0\right)$ holomorphe intégrable. On suppose que la partie initiale $\operatorname{In}(\omega)=\omega_{\nu}$ est dicritique et satisfait Cod Sing $\omega_{\nu} \geq 2$. Alors :

(1) Si $\nu=1, \mathcal{F}_{\omega}$ est holomorphiquement conjugué à $\mathcal{F}_{\omega_{1}}, \omega_{1}=z_{2} d z_{1}-z_{1} d z_{2}$; en particulier $\mathcal{F}_{\omega}$ possède intégrale première méromorphe $\frac{Z_{1}}{Z_{2}}\left(Z_{i}\right.$ coordonnées).

(2) Si $\nu=2, \mathcal{F}_{\omega}$ est donné par une 1-forme fermée méromorphe.

(3) Si $\nu \geq 3$ et $\left[\mathcal{F}_{\omega_{\nu}}\right]$ n'a ni singularité nilpotente, ni centre, alors $\mathcal{F}_{\omega}$ est conjugué à $\mathcal{F}_{\omega_{\nu}}$.

(4) Si $\nu=3$ et $\left[\mathcal{F}_{\omega_{3}}\right]$ a une singularité de type centre, alors $\mathcal{F}_{\omega}$ est défini par une 1 -forme fermée méromorphe.

(5) Si $\nu=3$ et $\left[\mathcal{F}_{\omega_{3}}\right]$ a une singularité nilpotente alors, modulo une condition de non résonnance portant sur $\omega_{3}, \mathcal{F}_{\omega}$ est défini par une 1 -forme fermée méromorphe ou bien $\mathcal{F}_{\omega}$ est conjugué à $\mathcal{F}_{\omega_{3}}$.

(6) Si $\nu=3$ et $\mathcal{F}_{\omega_{3}}$ a une singularité quadratique (1-jet nul), alors $\mathcal{F}_{\omega}$ est transversalement affine, i.e. $d \omega=\omega \wedge \omega_{1}$, avec $\omega_{1}$ méromorphe fermée.

Dans le Théorème $\mathrm{A}$, nous avons rassemblé divers énoncés qui apparaissent dans le texte. Par souci de cohérence nous avons inséré dans le théorème des résultats bien connus : c'est le cas des points 1 et 2. Le point 3 contient le résultat de Camacho-Lins Neto [1]. Le point 4 fait intervenir un résultat d'intégration Liouvillienne remarquable démontré en 1908 par Henri Dulac [14]. Le point 5 nécessite une étude fine des déploiements des feuilletages du plan à singularité nilpotente. Cette étude est rendue possible par l'utilisation du Théorème de Préparation de F. Loray [19. A titre d'exemple, on démontre que si $\omega_{0} \in \Omega^{1}\left(\mathbb{C}^{2}, 0\right)$ est à singularité nilpotente et à nombre de Milnor $\mu\left(\omega_{0} ; 0\right)$ de type $p-1$ avec $p$ premier, alors tout déploiement $\mathcal{F}_{\omega}$ de $\mathcal{F}_{\omega_{0}}$ à singularité nilpotente $\left(\operatorname{In}(\omega)=\operatorname{In}\left(\omega_{0}\right)\right)$ est trivial ou bien $\mathcal{F}_{\omega_{0}}\left(\right.$ et $\left.\mathcal{F}_{\omega}\right)$ possède une intégrale première holomorphe non constante. Rappelons que si $\omega_{0}=a d x+b d y$ alors $\mu\left(\omega_{0} ; 0\right)$ est la multiplicité d'intersection de l'idéal $(a, b)$ :

$$
\mu\left(\omega_{0} ; 0\right)=\operatorname{dim} \frac{\mathcal{O}\left(\mathbb{C}^{2}, 0\right)}{(a, b)} .
$$

Nous donnons des exemples de déploiements non triviaux pour les feuilletages $\mathcal{F}_{\omega_{3}}$ associés à des 1 -formes fermées méromorphes. En particulier on rencontre dans cette description des feuilletages $\mathcal{F}_{\omega}$, $\omega \in \Omega^{1}\left(\mathbb{C}^{3}, 0\right)$, qui après réduction des singularités sont complétement réguliers.

Remerciements. Nous remercions F. Loray, C. Rousseau et F. Touzet pour des échanges fructueux. Le deuxième auteur a bénéficié d'un financement IUF et CNPq pour un séjour à Rennes, le troisième est financé par un projet $\mathrm{PDE} / \mathrm{CsF}$ du CNPq pour un séjour de deux ans à Rennes.

\section{Notations, DÉFINitions ET RAPPELS}

On note $\mathcal{O}\left(\mathbb{C}^{n}, 0\right)$ l'anneau des germes de fonctions holomorphes à l'origine de $\mathbb{C}^{n}$; on désigne par $\Omega^{k}\left(\mathbb{C}^{n}, 0\right)$ le $\mathcal{O}\left(\mathbb{C}^{n}, 0\right)$-module des germes de $k$-formes holomorphes à l'origine de $\mathbb{C}^{n}$. Bien sûr $\Omega^{0}\left(\mathbb{C}^{n}, 0\right)=\mathcal{O}\left(\mathbb{C}^{n}, 0\right) ;$ le $\mathcal{O}\left(\mathbb{C}^{n}, 0\right)$-module des champs de vecteurs holomorphes se note $\Theta\left(\mathbb{C}^{n}, 0\right)$. Plus généralement si $M$ est une variété complexe on note $\mathcal{O}(M), \Omega^{k}(M)$ et $\Theta(M)$ les faiseaux de 
fonctions, $k$-formes et champs. Si $\alpha \in \Omega^{k}$ et $X \in \Theta$ on note $i_{X} \alpha$ le produit intérieur de $\alpha$ par $X$ et par $L_{X} \alpha=i_{X} d \alpha+d\left(i_{X} \alpha\right)$ la dérivée de Lie de $\alpha$ suivant $X$.

Un germe de feuilletage holomorphe $\mathcal{F}=\mathcal{F}_{\omega}$ de codimension 1 à l'origine de $\mathbb{C}^{n}$ est défini par la donnée d'un germe de 1 -forme $\omega \in \Omega^{1}\left(\mathbb{C}^{n}, 0\right)$ satisfaisant les deux conditions :

(i) $\omega \wedge d \omega=0$ (intégrabilité)

(ii) Cod Sing $\omega \geq 2$

où Sing $\omega$ désigne l'ensemble singulier de $\omega$; en coordonnées si :

$$
\omega=\sum_{i=1}^{n} a_{i} d x_{i}, a_{i} \in \mathcal{O}\left(\mathbb{C}^{n}, 0\right)
$$

alors Sing $\omega=\left\{a_{1}=\cdots=a_{n}=0\right\}$.

Ajoutons que par définition deux 1-formes $\omega, \omega^{\prime}$ satisfaisant (i), (ii) et $\omega \wedge \omega^{\prime}=0$ définissent le même feuilletage. Avec le notation ci-dessus le lieu singulier Sing $\mathcal{F}$ de $\mathcal{F}=\mathcal{F}_{\omega}$ est Sing $\omega$. Deux germes de feuilletages $\mathcal{F}_{\omega}=\mathcal{F}$ et $\mathcal{F}_{\omega^{\prime}}=\mathcal{F}^{\prime}$ sont conjugués s'il existe $\phi \in \operatorname{Diff}\left(\mathbb{C}^{\mathrm{n}}, 0\right)$, le groupe des germes de difféomorphismes de $\mathbb{C}^{n}, 0$, tel que $\phi^{*} \omega \wedge \omega^{\prime}=0$. On note $\mathcal{F}^{\prime}=\phi^{-1} \mathcal{F}$.

On a le théorème de structure suivant :

Théorème 1. (Malgrange [21]) Soit $\omega$ comme ci-dessus; si Cod Sing $\omega \geq 3$ il existe une unité $g \in \mathcal{O}^{*}\left(\mathbb{C}^{n}, 0\right)$ et $f \in \mathcal{O}\left(\mathbb{C}^{n}, 0\right)$ tels que $\omega=g d f$.

Lorsque $\omega$ est non singulière, i.e. Sing $\omega=\emptyset$, on retrouve le Théorème de Frobenius classique. Dans ce cas, $f$ est une submersion.

Dans la théorie des singularités de feuilletage il y a un énoncé classique connu sous le nom de phénomène de Kupka-Reeb et qui est une application directe du Théorème de Darboux classifiant localement les 2-formes fermées. En voici l'énoncé :

Théorème 2. [17, 3] Soit $\mathcal{F}=\mathcal{F}_{\omega}$ un germe de feuilletage à l'origine de $\mathbb{C}^{n}$ défini par la 1 -forme intégrable $\omega$. On suppose que la différentielle $d \omega$ est non nulle à l'origine : $d \omega(0) \neq 0$. Il existe des coordonnées $\left(x_{1}, x_{2}, \ldots, x_{n}\right)$ telles que $\omega=A_{1}\left(x_{1}, x_{2}\right) d x_{1}+A_{2}\left(x_{1}, x_{2}\right) d x_{2}, A_{i} \in \mathcal{O}\left(\mathbb{C}^{2}, 0\right)$.

Sous les hypothèses du théorème, il existe une submersion $\varphi: \mathbb{C}^{n}, 0 \rightarrow \mathbb{C}^{2}, 0$ telle que $\mathcal{F}=\varphi^{-1}\left(\mathcal{F}_{0}\right)$ où $\mathcal{F}_{0}$ est un feuilletage sur $\mathbb{C}^{2}, 0$.

Si l'on souhaite décrire tous les feuilletages donnés par une 1-forme $\omega$ dont la partie linéaire est non triviale les deux énoncés précédents s'avèrent insuffisants. Ils doivent être complétés par deux autres; le premier se trouve dans [12, nous en donnons sa preuve dont l'esprit est important pour la compréhension de la suite :

Proposition 1. Soit $\mathcal{F}$ un germe de feuilletage holomorphe de codimension 1 à l'origine de $\mathbb{C}^{n}$ donné par $\omega \in \Omega^{1}\left(\mathbb{C}^{n}, 0\right), n \geq 3$. Soient $i: \mathbb{C}^{2}, 0 \hookrightarrow \mathbb{C}^{n}, 0$ une immersion et $\omega_{0}=i^{*} \omega$. On suppose que le $1-j e t$ de $\omega_{0}$ est non nilpotent (i.e. non nul et non conjugué à $x_{1} d x_{1}$ ). On a l'alternative :

(1) $\mathcal{F}$ ne dépend que de deux variables, i.e. il existe une submersion $j: \mathbb{C}^{n}, 0 \rightarrow \mathbb{C}^{2}, 0, j \circ i=\mathrm{id}_{\mathbb{C}^{2}}$, telle que $\mathcal{F}=j^{-1}\left(i^{-1} \mathcal{F}\right)$.

(2) $\mathcal{F}$ possède une intégrale première holomorphe $f \in \mathcal{O}\left(\mathbb{C}^{n}, 0\right)$ non constante, i.e. $\omega \wedge d f=0$.

Démonstration. Soit $(x, y)=\left(x_{1}, x_{2}, y_{3}, \ldots, y_{n}\right)$ un système de coordonnées tel que $i$ s'identifie à l'inclusion $i(x)=(x, 0)$. Si $d \omega_{0}(0) \neq 0$ on a bien sûr $d \omega(0) \neq 0$ et on se trouve en présence d'un phénomène de Kupka-Reeb; on est alors dans le cas (1). Sinon, puisque le 1-jet de $\omega_{0}$ est non nilpotent, on a à conjugaison linéaire près :

$$
j^{1} \omega_{0}=x_{2} d x_{1}+x_{1} d x_{2} .
$$

On écrit alors :

$$
\omega=A_{1} d x_{1}+A_{2} d x_{2}+B_{3} d y_{3}+\cdots+B_{n} d y_{n}
$$

où $A_{i}, B_{i} \in \mathcal{O}\left(\mathbb{C}^{n}, 0\right)$ avec $A_{1}=x_{2}+\cdots, A_{2}=x_{1}+\cdots$. Si le lieu singulier de $\omega$ est de codimension au moins trois, le Théorème de Malgrange dit que nous sommes dans l'éventualité (2). Comme l'ensemble 
lisse $\left\{A_{1}=A_{2}=0\right\}$ est de codimension 2 et contient Sing $\omega$, on constate, lorsque Cod Sing $\omega=2$, l'égalité Sing $\omega=\left\{A_{1}=A_{2}=0\right\}$. En particulier chaque $B_{i}$ est dans l'idéal $<A_{1}, A_{2}>$ :

$$
B_{i}=\alpha_{i} A_{1}+\beta_{i} A_{2}, \quad \alpha_{i}, \beta_{i} \in \mathcal{O}\left(\mathbb{C}^{n}, 0\right)
$$

Par suite les champs de vecteurs $X_{j}=\frac{\partial}{\partial y_{j}}-\alpha_{j} \frac{\partial}{\partial x_{1}}-\beta_{j} \frac{\partial}{\partial x_{2}}$ annulent $\omega$ par produit intérieur et produisent une trivialisation de $\mathcal{F}$ : nous sommes dans le cas (1).

La description des feuilletages donnés par une 1-forme dont le 1-jet est $x_{1} d x_{1}$ est présentée par F. Loray dans [19] comme conséquence de son Théorème de Préparation. En voici l'énoncé :

Théorème 3. Soit $\mathcal{F}=\mathcal{F}_{\omega}$ un germe de feuilletage de codimension 1 à l'origine de $\mathbb{C}^{n}$. On suppose que la partie linéaire de $\omega$ est de type $x_{1} d x_{1}$. Alors à conjugaison et multiplication par unité près $\omega$ est de la forme :

$$
\omega=x_{1} d x_{1}+\left(l_{1}(f)+x_{1} l_{2}(f)\right) d f
$$

où $f=f\left(x_{2}, \ldots, x_{n}\right) \in \mathcal{O}\left(\mathbb{C}^{n-1}, 0\right), l_{1}$ et $l_{2}$ sont dans $\mathcal{O}(\mathbb{C}, 0)$.

Une 1-forme de type (1) sera appelée forme normale de Loray. Comme dans le phénomène de Kupka-Reeb, le feuilletage $\mathcal{F}$ apparait comme image réciproque (par l'application $\left(x_{1}, f\right)$ ) d'un feuilletage de $\mathbb{C}^{2}, 0$ (le feuilletage défini par $\left.x_{1} d x_{1}+\left(l_{1}\left(y_{1}\right)+x_{1} l_{2}\left(y_{1}\right)\right) d y_{1}\right)$.

Soit $\omega$ un germe de 1 -forme intégrable à l'origine de $\mathbb{C}^{n}$; on note $\nu=\nu(\omega)$ la multiplicité algébrique de $\omega$. Si on effectue le développement de Taylor de $\omega$ :

$$
\omega=\omega_{\nu}+\omega_{\nu+1}+\cdots+\omega_{k}+\cdots
$$

où chaque $\omega_{k}$ est une 1 -forme homogène de degré $k$, alors $\omega_{\nu}$ est une 1 -forme homogène qui se trouve intégrable (condition (i)); par contre la condition (ii) pour $\omega$, i.e. Cod Sing $\omega \geq 2$ n'implique pas qu'elle soit vérifiée par $\omega_{\nu}$.

Définition 1. Soit $\omega_{\nu}$ une 1-forme homogène intégrable sur $\mathbb{C}^{n}, \omega_{\nu}=\sum_{i=1}^{n} A_{i} d x_{i}$, $A_{i}$ homogène de degré $\nu$. On dit que $\omega_{\nu}$ est dicritique si $\sum_{i=1}^{n} x_{i} A_{i} \equiv 0$ et non dicritique sinon.

Considérons le champ radial $R=\sum_{i=1}^{n} x_{i} \frac{\partial}{\partial x_{i}} ; \omega_{\nu}$ est dicritique si et seulement si le polynôme $P_{\nu+1}=$ $i_{R} \omega_{\nu}$ est identiquement nul. Dans le cas non dicritique, $P_{\nu+1} \not \equiv 0$, la 1 -forme $\frac{\omega_{\nu}}{P_{\nu+1}}$ est fermée; si la décomposition en facteurs irréductibles de $P_{\nu+1}$ est :

$$
P_{\nu+1}=P_{1}^{n_{1}+1} \cdots P_{s}^{n_{s}+1}, \quad n_{i} \in \mathbb{N}, P_{i} \text { irréductible, }
$$

alors il existe des nombres $\lambda_{i} \in \mathbb{C}$ et un polynôme $H$ de degré $d^{0} H=\sum n_{i} d^{0} P_{i}$ tel que ([12]) :

$$
\frac{\omega_{\nu}}{P_{\nu+1}}=\sum \lambda_{i} \frac{d P_{i}}{P_{i}}+d\left(\frac{H}{P_{1}^{n_{1}} \cdots P_{s}^{n_{s}}}\right) \text {. }
$$

Cette 1 -forme possède l'intégrale première multivaluée $\sum \lambda_{i} \log P_{i}+\frac{H}{P_{1}^{n_{1}} \cdots P_{s}^{n_{s}}}$.

Lorsque $P_{\nu+1}$ est réduit, i.e. $n_{i}=0$, on peut supposer $H=0$ et :

$$
\frac{\omega_{\nu}}{P_{\nu+1}}=\sum \lambda_{i} \frac{d P_{i}}{P_{i}} \quad \text { avec } \sum \lambda_{i} d^{0} P_{i}=1
$$

Dans ce cas on dit que $\omega_{\nu}$ (ou $\frac{\omega_{\nu}}{P_{\nu+1}}$ ) est logarithmique et que les $\lambda_{i}$ sont les résidus de $\frac{\omega_{\nu}}{P_{\nu+1}}$.

On dit que les résidus satisfont la condition $\mathcal{P}$ si $\lambda_{i} \notin \mathbb{Q}$ pour tout $i$ et l'un des $\lambda_{i}$ est ou bien non réel ou bien un irrationnel mal approché par les rationnels.

Voici deux résultats dus à D. Cerveau et F. Loray [11] et D. Cerveau et J.-F. Mattei [12] que nous concentrons dans un seul et qui donnent un aperçu raisonnable des feuilletages $\mathcal{F}=\mathcal{F}_{\omega}$ lorsque $\omega_{\nu}$ est non dicritique logarithmique. 
Théorème 4. Soit $\omega=\omega_{\nu}+\cdots$ un germe de 1 -forme intégrable à l'origine de $\mathbb{C}^{n}, n \geq 3$. On suppose que la partie homogène de plus bas degré $\omega_{\nu}$ est non dicritique et satisfait Cod Sing $\omega_{\nu} \geq 2$.

(1) Si $P_{\nu+1}=i_{R} \omega_{\nu}$ est irréductible de degré $\nu+1=p^{s}$ avec $s \in \mathbb{N}$ et $p$ premier, alors $\omega$ possède une intégrale première holomorphe non constante $f=P_{\nu+1}+\cdots, \omega \wedge d f=0$.

(2) Si $P_{\nu+1}=P_{1} \cdots P_{s}$ est réduit, $s \geq 2$, l'hypersurface $\left[P_{\nu+1}=0\right] \subset \mathbb{P}_{\mathbb{C}}^{n-1}$ est à croisement ordinaires et les résidus $\lambda_{i}$ satisfont la condition $\mathcal{P}$, alors $\omega$ possède une intégrale première $\sum \lambda_{i} \log f_{i}$, où les $f_{i}=P_{i}+\cdots$ sont holomorphes.

(3) Si $P_{\nu+1}$ est irréductible et $\left[P_{\nu+1}=0\right] \subset \mathbb{P}_{\mathbb{C}}^{n-1}$ est à croisements ordinaires alors $\omega$ possède intégrale première holomorphe.

Remarque 1. Dans les cas (1), (2) et (3) le feuilletage $\mathcal{F}_{\omega}$ est défini par une 1-forme fermée tout comme sa partie homogène $\mathcal{F}_{\omega_{\nu}}$.

Remarque 2. Dans 11] on construit des feuilletages $\mathcal{F}_{\omega}$ de $\mathbb{C}^{3}, 0$ tels que la partie homogène $\omega_{\nu}$ soit de type $d P_{\nu+1}$ avec $P_{\nu+1}$ irréductible et qui ne possèdent pas d'intégrale première holomorphe non constante. Ils ont la propriété d'être image réciproque par une application holomorphe d'un feuilletage de $\mathbb{C}^{2}, 0$.

Remarque 3. Dans [13] on s'intéresse au cas où $P_{\nu+1}=0$ est un croisement normal, i.e. un produit de coordonnées, et les $\lambda_{i}$ des entiers positifs. On obtient dans ce cas l'alternative (non exclusive) : ou bien $\mathcal{F}_{\omega}$ est défini par une 1 -forme fermée (qui n'est pas en général à pôles simples) ou bien $\mathcal{F}_{\omega}$ est image récriproque d'un feuilletage de $\mathbb{C}^{2}, 0$.

Intéressons nous maintenant dans le cas dicritique aux propriétés de $\omega_{\nu}$ suceptibles d'être transmises à tout $\omega=\omega_{\nu}+\cdots$. Le premier résultat en ce sens est du à C. Camacho et A. Lins Neto [1] :

Théorème 5. (1) Soit $\omega=\omega_{\nu}+\cdots$ un germe de 1 -forme intégrable à l'origine de $\mathbb{C}^{3}$ dont le $\nu$-jet est la 1 -forme homogène dicritique $\omega_{\nu}$ avec $\nu \geq 3$. Si la 2 -forme $d \omega_{\nu}$ ne s'annule qu'en 0 , il existe un germe de difféomorphisme $\phi: \mathbb{C}^{3}, 0 \circlearrowleft$ tel que $\omega=\phi^{*} \omega_{\nu}$, i.e. $\omega$ et $\omega_{\nu}$ sont conjuguées.

(2) Soit $\omega \in \Omega^{1}\left(\mathbb{C}^{n}, 0\right), n \geq 4$, un germe de 1 -forme intégrable pour lequel il existe une section 3 -plane $i: \mathbb{C}^{3}, 0 \rightarrow \mathbb{C}^{n}, 0$ telle que $i^{*} \omega$ satisfait le point $(1)$. Alors il existe une submersion $j: \mathbb{C}^{n}, 0 \rightarrow \mathbb{C}^{3}, 0$ telle que $\omega=j^{*}\left(i^{*} \omega\right)$. En particulier $\omega$ est conjugué à la 1 -forme $\left(i^{*} \omega\right)_{\nu} \in \Omega^{1}\left(\mathbb{C}^{3}, 0\right)$ (vue dans $\left.\Omega^{1}\left(\mathbb{C}^{n}, 0\right)\right)$.

Le Théorème 5 classifie les feuilletages en dimension 3 à partie homogène générique pour $\nu \geq 3$. Qu'en est-il pour $\nu \leq 2$ ? Il se trouve que si $\omega_{\nu} \in \Omega^{1}\left(\mathbb{C}^{n}, 0\right)$ est intégrable homogène dicritique de degré $\nu \leq 2$, il existe un polynôme homogène $Q_{\nu+1}$ de degré $\nu+1$ tel que $\frac{\omega_{\nu}}{Q_{\nu+1}}$ soit fermée [3]. Par exemple si $\nu=1, \omega_{1}$ est linéairement conjugué à $x_{1} d x_{2}-x_{2} d x_{1}$ et $Q_{2}=x_{1} x_{2}$ convient.

On déduit la classification (générique lorsque) $\nu \leq 2$ en utilisant le résultat suivant énoncé dans [7] :

Théorème 6. Soit $\omega=\omega_{\nu}+\cdots \in \Omega^{1}\left(\mathbb{C}^{n}, 0\right)$ intégrable avec $\omega_{\nu}$ homogène dicritique de degré $\nu$ et satisfaisant $\operatorname{Cod} \operatorname{Sing} \omega_{\nu} \geq 2$. On suppose qu'il existe un polynôme homogène $Q$ tel que $\frac{\omega_{\nu}}{Q}$ soit fermée. Il existe alors $f \in \mathcal{O}\left(\mathbb{C}^{n}, 0\right)$ tel que $\frac{\omega}{f}$ soit fermée.

Remarque 4. De la même façon que dans le cas homogène, une 1-forme fermée méromorphe $\eta$ s'écrit :

$$
\eta=\sum_{i=1}^{s} \lambda_{i} \frac{d f_{i}}{f_{i}}+d\left(\frac{H}{f_{1}^{n_{1}} \cdots f_{s}^{n_{s}}}\right)
$$

où les $f_{i}$ et $H$ sont holomorphes, $\lambda_{i} \in \mathbb{C}$ et $n_{i} \in \mathbb{N}$. La fonction multivaluée $\sum \lambda_{i} \log f_{i}+\frac{H}{f_{1}^{n_{1}} \cdots f_{s}^{n_{s}}}$ est une intégrale première du feuilletage correspondant. 
Corollaire 1. Soit $\omega=\omega_{\nu}+\cdots \in \Omega^{1}\left(\mathbb{C}^{n}, 0\right)$ intégrable avec $\omega_{\nu}$ homogène dicritique, Cod Sing $\omega_{\nu} \geq 2$ et $\nu \leq 2$. Il existe $f \in \mathcal{O}\left(\mathbb{C}^{n}, 0\right)$ tel que $\frac{\omega}{f}$ soit fermée.

Remarque 5. Le Théorème 6 s'étend stricto-sensu (avec une démonstration différente) de la façon suivante : s'il existe $Q$ rationelle homogène telle que $\frac{\omega_{\nu}}{Q}$ soit fermée alors il existe $f$ méromorphe telle que $\frac{\omega}{f}$ soit fermée.

Remarque 6. En dimension supérieur à 4 l'espace des formes intégrables homogènes dicritiques de degré $\nu$ est une variété algébrique non irréductible. "Beaucoup" de ses composantes sont associés à des feuilletages définis par des 1 -formes fermées rationelles. C'est un cas typique où le Théorème 6 s'applique.

Ceci étant dit il semble intéressant d'étudier les dégénérescences des cas génériques - ce que nous abordons dans les chapitres suivants.

\section{CAS DiCRitique DÉGÉnÉRÉ AVEC $\nu \leq 2$}

Dans le paragraphe qui précède l'hypothèse Cod Sing $\omega_{\nu} \geq 2$ a été essentielle. En fait si $\omega_{\nu}$ est homogène dicritique avec $\nu \leq 2$ et $\omega_{\nu}$ s'annule sur une hypersurface alors $\nu=2$ et à conjugaison linéaire près $\omega_{2}$ est de l'un des deux types suivants :

$$
x_{1}\left(x_{1} d x_{2}-x_{2} d x_{1}\right) \quad \text { ou } \quad x_{3}\left(x_{1} d x_{2}-x_{2} d x_{1}\right) .
$$

Il existe des 1 -formes holomorphes $\omega \in \Omega^{1}\left(\mathbb{C}^{n}, 0\right)$ dont le 2 -jet est $x_{1}\left(x_{1} d x_{2}-x_{2} d x_{1}\right)$ pour lesquels il n'existe pas de $f \in \mathcal{O}\left(\mathbb{C}^{2}, 0\right)$ tels que $\frac{\omega}{f}$ soit fermée : c'est d'ailleurs générique par la topologie de Krull. Pour ce qui concerne les dimensions supérieures à 2 on peut poser la

Question : Soit $\omega \in \Omega^{1}\left(\mathbb{C}^{n}, 0\right)$ intégrable dont le 2-jet est $x_{1}\left(x_{1} d x_{2}-x_{2} d x_{1}\right)$. Existe-t-il $F$ : $\mathbb{C}^{n}, 0 \rightarrow \mathbb{C}^{2}, 0$ et $\omega_{0} \in \Omega^{1}\left(\mathbb{C}^{2}, 0\right)$ tels que $\omega \wedge F^{*} \omega_{0}=0$, i.e. le feuilletage $\mathcal{F}_{\omega}$ est-il le tiré en arrière d'un feuilletage du plan?

Pour ce qui concerne l'autre modèle nous avons la :

Proposition 2. Il n'existe pas de 1-forme $\omega \in \Omega^{1}\left(\mathbb{C}^{n}, 0\right), n \geq 3$, $\omega$ intégrable, Cod Sing $\omega \geq 2$ ayant pour $2-$ jet $\omega_{2}=x_{3}\left(x_{1} d x_{2}-x_{2} d x_{1}\right)$.

Démonstration. Quitte à couper par un 3-plan général, il suffit d'établir la proposition pour $n=3$. Supposons qu'il existe $\omega \in \Omega^{1}\left(\mathbb{C}^{3}, 0\right)$ telle que $j^{2} \omega=\omega_{2}$ et Cod Sing $\omega \geq 2$.

Considérons l'éclatement $E: \tilde{\mathbb{C}}^{3} \rightarrow \mathbb{C}^{3}, 0$ de l'origine de $\mathbb{C}^{3}$. Le diviseur exceptionnel $E^{-1}(0) \simeq \mathbb{P}_{\mathbb{C}}^{2}$ est génériquement transverse au feuilletage $\tilde{\mathcal{F}}$ relevé de $\mathcal{F}=\mathcal{F}_{\omega}$ par $E$. En se plaçant dans la carte $\left(x_{1}, x_{2}, x_{3}\right)$ où $E$ s'exprime sous la forme :

$$
E\left(x_{1}, x_{2}, x_{3}\right)=\left(x_{1}, x_{1} x_{2}, x_{1} x_{3}\right)
$$

on constate que $\tilde{\mathcal{F}}$ est défini par :

$$
\frac{E^{*} \omega}{x_{1}^{3}}=\tilde{\omega}=x_{3} d x_{2}+\tilde{A} d x_{1}+x_{1} \tilde{B} d x_{2}+x_{1} \tilde{C} d x_{3} .
$$

En écrivant explicitement l'intégrabilité de $\tilde{\omega}$ on constate que $\tilde{\omega}$ s'annule sur la droite $x_{1}=x_{3}=0$ contenue dans $E^{-1}(0)$. Un calcul immédiat montre que :

$$
\left.d \tilde{\omega}\right|_{x_{1}=x_{3}=0}=d x_{3} \wedge d x_{2}+d x_{1} \wedge(\cdot)
$$

Ainsi $\tilde{\omega}$ annule un champ $\tilde{Z}$ dont la composant suivant $\frac{\partial}{\partial x_{1}}$ est non nulle le long de $x_{1}=x_{3}=0$. Ceci oblige $\tilde{\omega}$ à s'annuler sur une surface, contredisant l'hypothèse Cod Sing $\omega \geq 2$. 
Si l'on considère l'espace $\mathcal{J}_{\nu} \subset \Omega^{1}\left(\mathbb{C}^{n}, 0\right)$ des germes de 1 -formes intégrables $\omega$ tels que $j^{\nu-1} \omega=0$, $\omega_{\nu}=j^{\nu} \omega$ est homogène de degré $\nu$ dicritique, on hérite d'une projection (jet d'ordre $\nu$ )

$$
j^{\nu}: \mathcal{J}_{\nu} \rightarrow\left[\mathcal{J}_{\nu}\right]
$$

à valeur dans $\left[\mathcal{J}_{\nu}\right]=\left\{\omega_{\nu}\right.$ homogène intégrable dicritique de degré $\left.\nu\right\}$. La Proposition 2 indique que cette projection n'est en général pas surjective.

Remarque 7. Le groupe des difféomorphismes tangents à l'identité $\operatorname{Diff}_{1}\left(\mathbb{C}^{n}, 0\right)$ agit sur $\mathcal{J}_{\nu}$ en préservant les fibres de $j^{\nu}$. Le Théorème 5 de Camacho-Lins Neto s'interprète comme suit : si $\underline{\omega}_{\nu}$ satisfait les hypothèses du Théorème 5 alors l'action de $\operatorname{Diff}_{1}\left(\mathbb{C}^{n}, 0\right)$ sur la fibre $\left(j^{\nu}\right)^{-1}\left(\underline{\omega}_{\nu}\right)$ est transitive.

\section{Un lemme utile et le Théorème de Camacho-Lins Neto Revisité}

Dans ce chapitre $\omega=\omega_{\nu}+\cdots \in \Omega^{1}\left(\mathbb{C}^{3}, 0\right)$ est un germe de 1 -forme intégrable, Cod Sing $\omega \geq 2$. Ils nous sera utile de réprésenter les germes considérés sur une boule centrée à l'origine. Nous la noterons $B$ et son rayon sera supposé suffisament petit pour que le discours qui suit ait un sens. On pose $B^{*}=B-\{0\}$.

Lemme 1. Supposons qu'il existe un recouvrement ouvert $\left\{U_{k}\right\}$ de $B^{*}$ et des champs de vecteurs holomorphes $X_{k} \in \Theta\left(U_{k}\right)$ tels que $\left.\omega\right|_{U_{k}}=\left.i_{X_{k}} d \omega\right|_{U_{k}}$. Il existe alors $X \in \Theta\left(\mathbb{C}^{3}, 0\right)$ tel que $\omega=i_{X} d \omega$.

Démonstration. Soit $Z$ un champ de vecteurs tel que $d \omega=i_{Z}$ vol où vol $=d x_{1} \wedge d x_{2} \wedge d x_{3}$ est une 3 -forme non nulle de $\mathbb{C}^{3}$. L'hypothèse du lemme implique que l'ensemble des zéros Sing $Z$ de $Z$ est contenu dans Sing $\omega$ et donc de codimension au moins deux. Lorsque $U_{i} \cap U_{j} \neq \emptyset$, on a $\left.i_{X_{i}-X_{j}} d \omega\right|_{U_{i} \cap U_{j}}=0$; puisque Cod Sing $Z \geq 2$ il existe $h_{i j} \in \mathcal{O}\left(U_{i} \cap U_{j}\right)$ tels que $X_{i}-X_{j}=h_{i j} Z$. Comme le groupe de cohomologie $H^{1}\left(B^{*}, \mathcal{O}\right)$ est trivial, c'est un résultat de H. Cartan [5] (voir [3]), on a, quitte à choisir un bon recouvrement, $h_{i j}=h_{i}-h_{j}, h_{k} \in \mathcal{O}\left(U_{k}\right)$. Ainsi les champs $X_{i}-h_{i} Z$ se recollent et définissent un champ global $X^{*} \in \Theta\left(B^{*}\right)$. Le Théorème de Hartogs permet de prolonger ce champ en un champ $X \in \Theta(B)$ qui vérifie $\omega=i_{X} d \omega$.

À titre d'application nous présentons une preuve de l'énoncé de Camacho-Lins Neto, partie (1).

Démonstration du Théorème 5, partie (1). Puisque $d \omega_{\nu}$ ne s'annule qu'en 0 il en est de même pour $d \omega$. Par suite en chaque point $m$ d'une boule épointée $B^{*}$ on peut trouver un champ $X_{, m} \in \Theta\left(B^{*}, m\right)$ tel que $\omega_{, m}=i_{X, m} d \omega_{, m}$. D'après le Lemme 1 il existe $X \in \Theta\left(\mathbb{C}^{3}, 0\right)$ tel que $\omega=i_{X} d \omega$. Il est clair que $X$ s'annule en 0 sinon $d \omega_{\nu}$ s'annulerait sur une droite. Soit $X_{1}$ la partie linéaire de $X$ en 0 ; on a:

$$
\omega_{\nu}=i_{X_{1}} d \omega_{\nu}=i_{\frac{R}{\nu+1}} d \omega_{\nu}
$$

qui conduit à $i_{X_{1}-\frac{R}{\nu+1}} d \omega_{\nu}=0$. Comme $\nu \geq 3$ et $d \omega_{\nu}$ est à singularité isolée on a $X_{1}=\frac{R}{\nu+1}$. Le Théorème de linéarisation des champs de vecteur de Poincaré permet alors de supposer que $X=X_{1}=\frac{R}{\nu+1}$. Un calcul direct (utilisant $L_{\frac{R}{\nu+1}} \omega=\omega$ ) montre que $\omega=\omega_{\nu}$.

Remarque 8. Dans l'article [1] les auteurs substituent au Lemme 1 le Lemme de Division de de Rham-Saito [25]. Il se trouve que le Lemme de Division peut se démontrer (sur $\mathbb{C}$ et en dimension 3) en utilisant l'argument de H. Cartan.

Remarque 9. Dans [18] l'un de nous montre que la seule hypothèse Sing $d \omega=\{0\}$ conduit, à conjugaison près, à l'existence d'un champ de vecteurs radial à poids $X=\sum p_{i} x_{i} \frac{\partial}{\partial x_{i}}, p_{i} \in \mathbb{N}^{*}$, tel que $L_{X} \omega=p \omega, p \in \mathbb{N}$, ce qui permet de montrer que $\omega$ est quasi-homogèneisable. Le feuilletage $\mathcal{F}_{\omega}$ peut être vue comme image réciproque d'un feuilletage sur un espace projectif à poids de dimension 2 .

Le lemme qui suit va nous permettre de donner une généralisation des résultats qui précèdent. Comme toujours on désigne par $E: \widetilde{\mathbb{C}}^{3} \rightarrow \mathbb{C}^{3}, 0$ l'éclatement de l'origine de $\mathbb{C}^{3} ;$ le diviseur exceptionnel $E^{-1}(0)$ est isomorphe à $\mathbb{P}_{\mathbb{C}}^{2}$. 
Lemme 2. Soit $\omega \in \Omega^{1}\left(\mathbb{C}^{3}, 0\right)$ un germe de 1 -forme intégrable à l'origine de $\mathbb{C}^{3}$ dont la partie homogène $\omega_{\nu}$ est dicritique et satisfait $\operatorname{Cod} \operatorname{Sing} \omega_{\nu} \geq 2$. On suppose qu'en chaque point $m \in E^{-1}(0)$ il existe un germe de champ de vecteur $\tilde{X}_{, m} \in \Theta\left(\tilde{\mathbb{C}}^{3}, m\right)$ tel que :

(1) $\tilde{X}_{, m}$ est tangent au feuilletage $E^{-1}\left(\mathcal{F}_{\omega}\right)$, i.e. $i_{\tilde{X}_{, m}} E^{*} \omega=0$.

(2) $\tilde{X}_{, m}$ est transverse $\grave{a} E^{-1}(0)$ en tout point $m \in E^{-1}(0)$.

Alors il existe $X \in \Theta\left(\mathbb{C}^{3}, 0\right)$ tel que $i_{X} d \omega=\omega=L_{X} \omega$.

Démonstration. Nous allons montrer que les hypothèses du Lemme 1 sont satisfaites. On écrit

$$
\omega=\omega_{\nu}+\omega_{\nu+1}+\cdots=\left(A_{\nu}+\cdots\right) d x_{1}+\left(B_{\nu}+\cdots\right) d x_{2}+\left(C_{\nu}+\cdots\right) d x_{3}
$$

où les $A_{\nu}, B_{\nu}, C_{\nu}$ sont homogènes de degré $\nu$ et satisfont :

$$
x_{1} A_{\nu}+x_{2} B_{\nu}+x_{3} C_{\nu}=0 .
$$

Plaçons nous dans la carte $\left(x_{1}, x_{2}, x_{3}\right)$ de $\tilde{\mathbb{C}}^{3}$ où l'expression de $E$ est donnée par $E\left(x_{1}, x_{2}, x_{3}\right)=$ $\left(x_{3} x_{1}, x_{3} x_{2}, x_{3}\right)$. Dans cette carte $E^{-1}(0)$ est donné par $\left(x_{3}=0\right)$ et

$$
E^{*} \omega=x_{3}^{\nu+1}\left[\left(A_{\nu}\left(x_{1}, x_{2}, 1\right)+x_{3} A^{\prime}\right) d x_{1}+\left(B_{\nu}\left(x_{1}, x_{2}, 1\right)+x_{3} B^{\prime}\right) d x_{2}+C^{\prime} d x_{3}\right]
$$

où les $A^{\prime}, B^{\prime}, C^{\prime}$ sont holomorphes le long de $x_{3}=0$.

En chaque point $m \in E^{-1}(0)$, l'hypothèse implique l'existence d'un champ de vecteur $Y_{, m}$ :

$$
Y_{, m}=\frac{\partial}{\partial x_{3}}+Y_{1} \frac{\partial}{\partial x_{1}}+Y_{2} \frac{\partial}{\partial x_{2}}, \quad Y_{i} \text { holomorphes, }
$$

tel que $i_{Y_{, m}} E^{*} \omega_{, m}=0$. En conjugant localement $Y_{, m}$ à $\frac{\partial}{\partial x_{3}}$ par un difféomorphisme local fibré $\phi_{, m}=$ $\left(\varphi, x_{3}\right), \varphi\left(x_{1}, x_{2}, 0\right)=\left(x_{1}, x_{2}\right)$, on conjugue $E^{*} \omega_{, m}$ à

$$
W x_{3}^{\nu+1}\left(A_{\nu}\left(x_{1}, x_{2}, 0\right) d x_{1}+B_{\nu}\left(x_{1}, x_{2}, 0\right) d x_{2}\right)
$$

où $W$ est une unité, $W\left(x_{1}, x_{2}, 0\right)=1$. Quitte à changer $x_{3}$ en $W^{\frac{1}{\nu+1}} x_{3}$, on conjugue $E^{*} \omega_{, m}$ à :

$$
\Omega=x_{3}^{\nu+1}\left(A_{\nu}\left(x_{1}, x_{2}, 0\right) d x_{1}+B_{\nu}\left(x_{1}, x_{2}, 0\right) d x_{2}\right) .
$$

Un calcul élémentaire montre que $i_{\frac{\partial}{\partial x_{3}}} d \Omega=(\nu+1) \Omega$. De sorte que l'on peut modifier le champ $\tilde{X}_{, m}$ pour qu'il satisfasse $i_{\tilde{X}_{, m}} d E^{*} \omega_{, m}=E^{*} \omega_{, m}$. La collection des $\tilde{X}_{, m}$ nous permet de construire des champs $X_{k}$ satisfaisant le Lemme 1 .

Nous allons maintenant décrire une première conséquence du Lemme 2 . Une 1-forme dicritique $\omega_{\nu} \in \Omega^{1}\left(\mathbb{C}^{3}\right)$, Cod Sing $\omega_{\nu} \geq 2$, induit un feuilletage $\left[\mathcal{F}_{\omega_{\nu}}\right]$ de degré $\nu-1$ sur $\mathbb{P}_{\mathbb{C}}^{2}$. Ce feuilletage possède exactement $\nu^{2}-\nu+1$ points singuliers comptés avec multiplicité. Nous dirons que $\left[\mathcal{F}_{\omega_{\nu}}\right]$ est sans nilpotence si en tout point $m \in \operatorname{Sing}\left[\mathcal{F}_{\omega_{\nu}}\right]$ le feuilletage est défini par une 1 -forme locale $\alpha_{, m}$ dont la partie linéaire est non nilpotente (en particulier non nulle). Dit autrement $\alpha_{, m}$ est ou bien de type Kupka-Reeb, i.e. $d \alpha(m) \neq 0$, ou bien le 1 -jet de $\alpha$ en $m$ est à conjugaison près $d\left(x_{1} x_{2}\right)$. Dans ce dernier cas nous dirons que $m \in \operatorname{Sing}\left[\mathcal{F}_{\omega_{\nu}}\right]$ est de type centre ou central si $\left[\mathcal{F}_{\omega_{\nu}}\right]_{, m}$ possède une intégrale première non constante : $\alpha_{, m}=g d f, f \in \mathcal{O}\left(\mathbb{P}_{\mathbb{C}}^{2}, m\right), g \in \mathcal{O}^{*}\left(\mathbb{P}_{\mathbb{C}}^{2}, m\right)$. Notons qu'un tel $f$ est nécessairement de Morse en $m$.

Proposition 3. Soit $\omega=\omega_{\nu}+\cdots \in \Omega^{1}\left(\mathbb{C}^{3}, 0\right)$ un germe de 1 -forme intégrable à l'origine de $\mathbb{C}^{3}$ dont la partie homogène $\omega_{\nu}$ est dicritique, $\nu \geq 3$ et $\operatorname{Cod} \operatorname{Sing} \omega_{\nu} \geq 2$. Si $\left[\mathcal{F}_{\omega_{\nu}}\right]$ est sans nilpotence et n'a pas de point central, alors $\omega$ et $\omega_{\nu}$ sont conjugués; en particulier $\mathcal{F}_{\omega}$ et $\mathcal{F}_{\omega_{\nu}}$ sont conjugués.

Démonstration. D'après la Proposition 1 les hypothèses du Lemme 2 sont satisfaites. Il existe donc un champ $X \in \Theta\left(\mathbb{C}^{3}, 0\right)$ tel que $i_{X}(d \omega)=\omega$. On conclut comme dans la preuve du Théorème 5: comme $\omega_{\nu}=i_{\frac{R}{\nu+1}} d \omega_{\nu}$ et Cod Sing $\omega_{\nu} \geq 2$ on a Cod Sing $d \omega_{\nu} \geq 2$. Par suite si $X_{1}=j^{1} X$, l'égalité $i_{X_{1}-\frac{R}{\nu+1}} d \omega_{\nu}=0$ implique que $X_{1}=\frac{R}{\nu+1}$. On conclut en linéarisant $X$. 
Remarque 10. La condition sans nilpotence, sans point central est générique dans l'espace des 1 -formes dicritiques de degré $\nu$.

On peut interpréter la Proposition 3 comme un énoncé de rigidité ou de détermination finie.

Proposition 4. Soit $\omega=\omega_{\nu}+\cdots \in \Omega^{1}\left(\mathbb{C}^{3}, 0\right)$ un germe de 1 -forme intégrable à l'origine de $\mathbb{C}^{3}$ dont la partie homogène $\omega_{\nu}$ est dicritique, $\nu \geq 3$ et $\operatorname{Cod} \operatorname{Sing} \omega_{\nu} \geq 2$. On suppose qu'en chaque point singulier $m$ de $\left[\mathcal{F}_{\omega_{\nu}}\right]$ celui ci est donné par une $1-$ forme à 1 -jet non nul. Si Sing $\omega$ est homéomorphe $\grave{a}$ Sing $\omega_{\nu}$, alors $\omega$ et $\omega_{\nu}$ sont conjugués.

Démonstration. Laissée au lecteur.

\section{Feuilletages avec singularités nilpotentes; Problèmes de DÉPloiements}

Dans ce chapitre on identifie $\mathbb{C}^{3}$ à $\mathbb{C}^{2} \times \mathbb{C}$ et l'on considère un feuilletage $\mathcal{F}_{0}$ sur $\mathbb{C}^{2}, 0$ ayant une singularité nilpotente non triviale en 0 , i.e. avec 1 -jet nilpotent non nul. Soit $\mathcal{F}$ un déploiement de $\mathcal{F}_{0}$ sur $\mathbb{C}^{3}$, c'est à dire un germe de feuilletage en $0 \in \mathbb{C}^{3}$ dont la restriction à $\mathbb{C}^{2} \times\{0\}$ est $\mathcal{F}_{0}$. Si $\omega \in \Omega^{1}\left(\mathbb{C}^{3}, 0\right)$ est une 1 -forme définissant $\mathcal{F}$, le feuilletage $\mathcal{F}_{0}$ est défini par $\omega_{0} \in \Omega^{1}\left(\mathbb{C}^{2}, 0\right)$ restriction de $\omega$ à $\mathbb{C}^{2} \times\{0\}$. On fixe des coordonnées $\left(x_{1}, x_{2}, x_{3}\right)$ de $\mathbb{C}^{3}=\mathbb{C}^{2} \times \mathbb{C} ;$ comme $\mathcal{F}_{0}$ est à singularité nilpotente non triviale on peut supposer que le 1 -jet de $\omega_{0}$ est $x_{1} d x_{1}$. Il se pourrait que $\omega$ soit non singulière en 0 , c'est le cas par exemple pour le feuilletage par les niveaux de $x_{1}^{2}+x_{2}^{3}+x_{3}$; mais dans ce cas $\mathcal{F}$ et donc $\mathcal{F}_{0}$ possède une intégrale première holomorphe non constante (Frobenius classique).

Supposons maintenant $\omega$ singulière en 0 . Le $1-$ jet de $\omega$ est du type :

$$
\omega_{1}=x_{1} d x_{1}+x_{3} d l+L d x_{3}
$$

où $l=l\left(x_{1}, x_{2}\right)$ et $L=L\left(x_{1}, x_{2}, x_{3}\right)$ sont des formes linéaires. L'intégrabilité de $\omega$ impliquant celle de $\omega_{1}$ on a :

$$
\left(x_{1} d x_{1}+x_{3} d l\right) \wedge d(l-L) \wedge d x_{3} \equiv 0
$$

qui implique $d \omega(0)=d \omega_{1}(0)=\lambda d x_{1} \wedge d x_{3}, \lambda \in \mathbb{C}$.

Il y a deux cas suivant que $\lambda$ est nul ou non. Si $d \omega_{1}(0) \neq 0$ alors $l=l\left(x_{1}\right), L=L\left(x_{1}, x_{3}\right)$ et nous avons un phénomène de Kupka-Reeb : il existe une submersion

$$
F:\left(x_{1}, x_{2}, x_{3}\right) \rightarrow\left(X_{1}\left(x_{1}, x_{2}, x_{3}\right), X_{3}\left(x_{1}, x_{2}, x_{3}\right)\right), \quad F\left(x_{1}, 0, x_{3}\right)=\left(x_{1}, x_{3}\right),
$$

telle que $\omega=F^{*} \alpha$ où $\alpha$ est la restriction de $\omega$ au plan $x_{2}=0 . \mathrm{Si}$

$$
\omega=A d x_{1}+B d x_{2}+C d x_{3}=x_{1} d x_{1}+\cdots
$$

on a $F^{*} \alpha=A\left(X_{1}, 0, X_{3}\right) d X_{1}+C\left(X_{1}, 0, X_{3}\right) d X_{3}$. Ainsi $\omega_{0}$ est la restriction de $F^{*} \alpha$ au plan $x_{3}=0$. Un calcul direct élémentaire montre que le nombre de Milnor $\mu\left(\mathcal{F}_{0} ; 0\right)$ de $\mathcal{F}_{0}$ en 0 est plus grand ou égal à 3 . Dans ce cas il y a un facteur intégrant pour $\mathcal{F}$, et donc $\mathcal{F}_{0}$ (tout du moins formel).

Supposons maintenant $\omega_{1}$ fermée :

$$
\omega_{1}=d\left(\frac{x_{1}^{2}}{2}+x_{3} l+\varepsilon x_{3}^{2}\right)=d q, \quad \varepsilon \in \mathbb{C}, \quad l=l\left(x_{1}, x_{2}\right) \text { linéaire. }
$$

De nouveau nous distinguons trois cas suivant le rang de la forme quadratique $q$.

Si $q$ est de rang 3, la forme $\omega$ est à singularité isolée et les feuilletages $\mathcal{F}$ et donc $\mathcal{F}_{0}$ possèdent une intégrale première holomorphe non constante (Théorème de Malgrange).

Si $q$ est de rang $2, q=\frac{x_{1}^{2}}{2}+x_{3}\left(a x_{1}+\varepsilon x_{3}\right)$, alors d'après la Proposition 1 ou bien $\mathcal{F}$ et donc $\mathcal{F}_{0}$ possèdent une intégrale première holomorphe ou bien il existe une submersion $F: \mathbb{C}^{3}, 0 \rightarrow \mathbb{C}^{2}, 0=$ $\left(x_{2}=0\right)$,

$$
F:\left(x_{1}, x_{2}, x_{3}\right) \rightarrow\left(X_{1}\left(x_{1}, x_{2}, x_{3}\right), X_{3}\left(x_{1}, x_{2}, x_{3}\right)\right),
$$

$F\left(x_{1}, 0, x_{3}\right)=\left(x_{1}, x_{3}\right)$, dont les fibres sont tangents à $\mathcal{F}$. Avec les mêmes notations que précédemment le feuilletage $\mathcal{F}$ est donné par $A\left(X_{1}, 0, X_{3}\right) d X_{1}+C\left(X_{1}, 0, X_{3}\right) d X_{3}$ et $\mathcal{F}_{0}$ par $A\left(X_{1}, 0, X_{3}\right) d X_{1}+$ $\left.C\left(X_{1}, 0, X_{3}\right) d X_{3}\right|_{x_{3}=0}$. Comme $\mathcal{F}_{0}$ possède facteur intégrant formel, il en est de même pour $\mathcal{F}[12$, 13. Un calcul élémentaire montre que le nombre de Milnor de cette dernière forme est plus grand que trois. 
Avant d'attaquer le cas restant où $q$ est de rang 1 , nous résumons la situation dans la

Proposition 5. Soit $\mathcal{F}=\mathcal{F}_{\omega}$ un déploiement de $\mathcal{F}_{0}$ sur $\mathbb{C}^{3}, 0=\mathbb{C}^{2}, 0 \times \mathbb{C}, 0, \mathcal{F}_{0}=\left.\mathcal{F}\right|_{\mathbb{C}^{2}, 0 \times\{0\}}$. On suppose que $\mathcal{F}_{0}$ a une singularité nilpotente non triviale en 0 . On est dans l'un des cas suivants:

(1) $\mathcal{F}$ et $\mathcal{F}_{0}$ ont une intégrale première holomorphe non constante.

(2) Le nombre de Milnor $\mu\left(\mathcal{F}_{0} ; 0\right)$ est plus grand que 3 et $\mathcal{F}$ et $\mathcal{F}_{0}$ ont un facteur intégrant (formel).

(3) Le $1-j e t$ de $\omega$ en 0 est $\left(x_{1}+\delta x_{3}\right) d\left(x_{1}+\delta x_{3}\right), \delta \in \mathbb{C}$. À conjugaison linéaire près on peut supposer que $\delta=0$.

Le cas restant correspondant au cas (3) où $\operatorname{rang} q=1$ se traite de la façon suivante, tout de moins lorsque $\mu\left(\mathcal{F}_{0} ; 0\right)=2$.

Proposition 6. Soit $\mathcal{F}=\mathcal{F}_{\omega}$ un déploiement de $\mathcal{F}_{0}=\mathcal{F}_{\omega_{0}}=\left.\mathcal{F}\right|_{\mathbb{C}^{2}, 0 \times\{0\}}$; on suppose que le $1-$ jet de $\omega$ est $j^{1} \omega=x_{1} d x_{1}$. Si $\mu\left(\mathcal{F}_{0} ; 0\right)=2$, alors on est dans l'une des situations suivantes :

(1) $\mathcal{F}_{0}$ (et $\mathcal{F}$ ) ont une intégrale première holomorphe.

(2) Le déploiement $\mathcal{F}$ de $\mathcal{F}_{0}$ est trivial, plus précisément il existe un champ de vecteurs $\xi \in \Theta\left(\mathbb{C}^{3}, 0\right)$

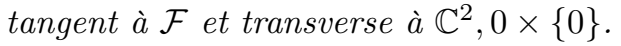

Démonstration. Elle repose sur la forme normale de Loray. En fait l'énoncé 3 dit que l'on peut supposer à unité multiplicative près que

$$
\omega=X_{1} d X_{1}+\left(l_{1}(f)+X_{1} l_{2}(f)\right) d f
$$

avec $f=f\left(x_{2}, x_{3}\right) \in \mathcal{O}\left(\mathbb{C}^{2}, 0\right), l_{1}$ et $l_{2} \in \mathcal{O}(\mathbb{C}, 0)$ et $X_{1}=x_{1}+\cdots \in \mathcal{O}\left(\mathbb{C}^{3}, 0\right)$.

Le feuilletage $\mathcal{F}_{0}$ est alors donné par

$$
\omega_{0}=X_{1}\left(x_{1}, x_{2}, 0\right) d X_{1}\left(x_{1}, x_{2}, 0\right)+\left(l_{1}\left(f\left(x_{2}, 0\right)\right)+X_{1} l_{2}\left(f\left(x_{2}, 0\right)\right)\right) d f\left(x_{2}, 0\right)
$$

qui est conjugué à

$$
\omega_{0}^{\prime}=x_{1} d x_{1}+\left(l_{1}\left(f_{0}\left(x_{2}\right)\right)+x_{1} l_{2}\left(f_{0}\left(x_{2}\right)\right)\right) d f_{0}\left(x_{2}\right)
$$

avec $f_{0}\left(x_{2}\right)=f\left(x_{2}, 0\right)$. Si $f_{0}$ est une submersion alors $(X, f)$ aussi et l'on est dans le cas $(2)$. On peut donc supposer que $f_{0}\left(x_{2}\right)=x_{2}^{k}$ avec $k \geq 2$.

Remarquons que si $l_{1}$ est une unité alors $\omega_{0}^{\prime}$ possède une intégrale première holomorphe et nous sommes dans le cas (1). En effet $\omega_{0}^{\prime}$ apparait comme image réciproque par l'application $\left(x_{1}, x_{2}^{k}\right)$ de

$$
\omega_{0}^{\prime \prime}=x_{1} d x_{1}+\left(l_{1}(u)+x_{1} l_{2}(u)\right) d u
$$

qui se trouve non singulière, donc avec intégrale première. On peut donc supposer que $l_{1}=\varepsilon u^{l}+\cdots$, $l \geq 1, \varepsilon \neq 0$; l'idéal des composantes de $\omega_{0}^{\prime}$ est donc $<x_{1}, x_{2}^{l k+k-1}>$. Comme $k \geq 2$ et $l \geq 1$, $k(l+1)-1 \geq 3$ et donc $\mu\left(\mathcal{F}_{0} ; 0\right) \geq 3$, cas exclus par hypothèse.

Dans [8] on classifie les feuilletages de degré 2 du plan $\mathbb{P}_{\mathbb{C}}^{2}$ n'ayant qu'une seule singularité. Il découle de cette classification que si $m$ est un point singulier de type nilpotent non trivial d'un feuilletage $\mathcal{F}_{0}$ de degré 2 alors $\mu\left(\mathcal{F}_{0} ; m\right) \leq 6$.

En fait la Proposition [ 6 s'étend comme suit :

Théorème 7. Soit $\mathcal{F}=\mathcal{F}_{\omega}$ un feuilletage de $\mathbb{C}^{3}, 0$ déploiement de $\mathcal{F}_{\omega_{0}}=\mathcal{F}_{0}=\left.\mathcal{F}\right|_{\mathbb{C}^{2}, 0 \times\{0\}} ;$ on suppose que le $1-$ jet de $\omega$ est $x_{1} d x_{1}$.

(1) Si $\mu\left(\mathcal{F}_{0} ; 0\right)=3$ alors ou bien le déploiement $\mathcal{F}$ est trivial ou bien $\mathcal{F}_{0}$ (et donc $\mathcal{F}$ ) possède un facteur intégrant formel $g_{0} \in \widehat{\mathcal{O}\left(\mathbb{C}^{2}, 0\right)}$ (respectivement $g \in \mathcal{O}\left(\mathbb{C}^{3}, 0\right)$ ) tel que $d\left(\frac{\omega_{0}}{g_{0}}\right)=0$ (respectivement $\left.d\left(\frac{\omega}{g}\right)=0\right)$.

(2) Si $\mu\left(\mathcal{F}_{0} ; 0\right)+1$ est un nombre premier alors ou bien le déploiement $\mathcal{F}$ est trivial ou bien $\mathcal{F}_{0}$ (et donc $\mathcal{F}$ ) possède une intégrale première holomorphe non constante. 
Démonstration. Elle procède comme la précédente; lorsque le déploiement est non trivial on a, avec les mêmes notations :

$$
\omega_{0}^{\prime}=x_{1} d x_{1}+\left(l_{1}\left(x_{2}^{k}\right)+x l_{2}\left(x_{2}^{k}\right)\right) k x_{2}^{k-1} d x_{2}, \quad k \geq 2 .
$$

Visiblement $\omega_{0}^{\prime}$ est image réciproque par $\left(x_{1}, x_{2}^{k}\right)$ de :

$$
\omega_{0}^{\prime \prime}=x_{1} d x_{1}+\left(l_{1}(u)+x_{1} l_{2}(u)\right) d u .
$$

Comme dans la preuve précédente si $l_{1}$ est unité $\omega_{0}^{\prime \prime}$ possède une intégrale première holomorphe et par suite $\mathcal{F}_{0}$ et $\mathcal{F}$ aussi. Sinon le 1 -jet de $\omega_{0}^{\prime \prime}$ est $x_{1} d x_{1}+\left(\varepsilon u+\lambda x_{1}\right) d u$ avec $\varepsilon=l_{1}^{\prime}(0)$ et $\lambda=l_{2}(0)$.

Ce 1 -jet est nilpotent si et seulement si $\varepsilon=\lambda=0$; s'il est non nilpotent, la théorie élémentaire des formes normales [12] assure que $\omega_{0}^{\prime \prime}$ (et donc $\omega_{0}^{\prime}$ et puis $\omega$ ) possède un facteur intégrant formel. On pose $l_{1}(u)=\varepsilon_{p} u^{p}+\cdots, \varepsilon_{p} \neq 0$, de sorte que

$$
l_{1}\left(x_{2}^{k}\right)=\varepsilon_{p} x_{2}^{k p}+\cdots, \quad k \geq 2 .
$$

L'idéal des composantes de $\omega_{0}^{\prime}$ est donc $<x_{1}, x_{2}^{k(p+1)-1}>$ et par suite $\mu\left(\mathcal{F}_{0} ; 0\right)+1=k(p+1)$. Comme $k \geq 2$, si $\mu\left(\mathcal{F}_{0} ; 0\right)+1$ est premier alors $p=0$ et $l_{1}$ est une unité; de sorte que $\mathcal{F}_{0}$ a une intégrale première holomorphe non constante. Lorsque $\mu\left(\mathcal{F}_{0} ; 0\right)=3$, alors ou bien $(k, p)=(4,0)$ et $\mathcal{F}_{0}$ a une intégrale première holomorphe, ou bien $(k, p)=(2,1)$, cas où $\mathcal{F}_{0}$ a un facteur intégrant formel.

Remarque 11. Comme on l'a dit, pour une singularité nilpotente d'un feuilletage de degré 2 on a que $\mu \in\{2,3,4,5,6\}$; seul le nombre 5 n'apparait pas dans la liste du Théorème 7 Dans ce cas avec les notations précédentes on a, lorsque $k \geq 2$, les possibilités $(k, p)=(6,0)$ (présence d'intégrale première holomorphe), $(k, p)=(3,1)$ (présence de facteur intégrant formel) mais aussi à priori $(k, p)=(2,2)$; dans ce cas, si $\lambda \neq 0$, il y a encore un facteur intégrant formel.

Remarque 12. Le cas $\mu=2$, traité dans la Proposition 6, l'est aussi dans le Théorème 7 . Nous avons souhaité séparer ces deux cas pour des raisons de lisibilité. Remarquons aussi que l'énoncé 7 se généralise en dimension $n$ quelconque avec une preuve identique.

Exemple : Soit $\mathcal{F}_{0}$ le feuilletage de degré 2 défini par $\omega_{0}=\left(x_{1}-x_{2}^{3}\right) d x_{1}+x_{1} x_{2}^{2} d x_{2}=\left(x_{1}-x_{2}^{3}\right) d x_{1}+$ $\frac{1}{3} x_{1} d x_{2}^{3}$. On a $\mu\left(\mathcal{F}_{0} ; 0\right)=5, k=3, p=1$ et $\mathcal{F}_{0}$ possède l'intégrale première $\frac{3 x_{1}-2 x_{2}^{3}}{x_{1}^{3}}$.

\section{Théorème de Dulac : PRÉSEntation et applications}

Rappelons un résultat célèbre d'Henri Dulac [14] sous sa forme généralisée dans [9] :

Théorème 8. Soit $\mathcal{F}$ un feuilletage de degré deux sur le plan projectif $\mathbb{P}_{\mathbb{C}}^{2}$. Supposons qu'il existe un point singulier $m \in \operatorname{Sing} \mathcal{F}$ de type centre. Alors $\mathcal{F}$ est défini par une 1 -forme fermée rationelle $\eta$.

En fait Dulac donne en carte affine une liste de formes normales pour les feuilletages de degré 2 ayant une singularité de type centre sous l'hypothèse que la droite à l'infini est invariante (il considère en effet des 1 -formes de type $A d x+B d y$ où $A$ et $B$ sont des polynômes de degré inférieur ou égal à 2). Dans [9] on montre que la présence d'une singularité de type centre implique celle d'une droite invariante, ce qui permet d'invoquer Dulac. Les preuves reposent sur des calculs formels longs typiques du début du 20e siècle et l'intuition d'un tel résultat reste mystérieuse; il serait intéressant d'ailleurs d'en produire une démonstration géométrique.

Ce théorème doit être interprété comme suit : une certaine condition d'intégrabilité locale (condition de centre) conduit à un résultat d'intégrabilité globale (primitive d'une forme fermée rationelle).

Voici la liste des modèles obtenus pas H. Dulac. Les feuilletages génériques ont une singularité de type centre. Par contre il y a des feuilletages qui n'ont pas de singularités centrales; ces deux situations sont contenues dans la liste ci-dessous.

Théorème 9. (Dulac [14]) La 1-forme fermée rationelle $\eta$ est d'un des types suivants :

(a) $\eta=d q$, où $q$ est un polynôme de degré 3 .

(b) $\eta=\sum_{i=1}^{3} \lambda_{j} \frac{d p_{j}}{p_{j}}$, où $\lambda_{j} \in \mathbb{C}^{*}$ et $p_{j}$ est un polynôme de degré $1,1 \leq j \leq 3$. 
(c) $\eta=\sum_{i=1}^{2} \lambda_{j} \frac{d p_{j}}{p_{j}}$, où $\lambda_{j} \in \mathbb{C}^{*}, j=1,2, \operatorname{deg}\left(p_{1}\right)=2$ et $\operatorname{deg}\left(p_{2}\right)=1$.

(d) $\eta=\sum_{i=1}^{2} \lambda_{j} \frac{d p_{j}}{p_{j}}+d q$, où $\lambda_{j} \in \mathbb{C}^{*}, \operatorname{deg}\left(p_{j}\right)=1, j=1,2$, et $\operatorname{deg}(q)=1$.

(e) $\eta=\sum_{i=1}^{2} \lambda_{j} \frac{d p_{j}}{p_{j}}+d\left(\frac{q}{p_{1}}\right)$, ò̀ $\lambda_{1}, \lambda_{2}, p_{1}, p_{2}, q$ sont comme en $(\mathrm{d})$.

(f) $\eta=\frac{d p}{p}+d\left(\frac{q}{p^{2}}\right)$, où $\operatorname{deg}(p)=1$ et $\operatorname{deg}(q)=2$.

(g) $\eta=\frac{d p}{p}+d\left(\frac{q}{p}\right)$, où $p$ et $q$ sont comme en (f).

(h) $\eta=\frac{d p}{p}+d q$, où $p$ et $q$ sont comme en (f).

(i) $\eta=\frac{d p}{p}+d q$, où $\operatorname{deg}(p)=2$ et $\operatorname{deg}(q)=1$.

(j) $\eta=3 \frac{d f}{f}-2 \frac{d g}{g}$, où $\operatorname{deg}(f)=2, \operatorname{deg}(g)=3$ et $3 g d f-2 f d g$ est divisible par une fonction affine.

Voici une conséquence du Théorème de Dulac :

Théorème 10. Soit $\omega=\omega_{3}+\cdots \in \Omega^{1}\left(\mathbb{C}^{3}, 0\right)$ un germe de 1 -forme intégrable à l'origine de $\mathbb{C}^{3}$ dont la partie homogène $\omega_{3}$ est dicritique et $\operatorname{Cod}$ Sing $\omega_{3} \geq 2$. Soit $\left[\mathcal{F}_{\omega_{3}}\right]$ le feuilletage de degré deux de $\mathbb{P}_{\mathbb{C}}^{2}$ induit par $\omega_{3}$.

(1) Si $\left[\mathcal{F}_{\omega_{3}}\right]$ possède un point central alors $\mathcal{F}_{\omega}$ est défini par une 1 -forme fermée méromorphe $\eta$.

(2) Si $\left[\mathcal{F}_{\omega_{3}}\right]$ ne possède pas de point singulier de type centre ou nilpotent, alors $\mathcal{F}_{\omega}$ est conjugué au feuilletage homogène $\mathcal{F}_{\omega_{3}}$.

Démonstration. Si $\left[\mathcal{F}_{\omega_{3}}\right]$ satisfait le point $(2)$ on applique la Proposition $3 \mathrm{Si}\left[\mathcal{F}_{\omega_{3}}\right]$ a un point central on invoque le Théorème de Dulac : $\left[\mathcal{F}_{\omega_{3}}\right]$ est défini par une 1 -forme fermée rationelle $\eta$. Soit $E: \tilde{\mathbb{C}}^{3}, 0 \rightarrow$ $\mathbb{C}^{3}, 0$ l'éclatement de l'origine. La restriction $\left.E^{*} \underline{\eta}\right|_{E^{-1}(0)}$ défini le feuilletage $\left[\mathcal{F}_{\omega_{3}}\right]$ sur $E^{-1}(0) \simeq \mathbb{P}_{\mathbb{C}}^{2}$. Le Théorème d'extension de Lévi permet de construire une 1-forme méromorphe $\tilde{\eta}$ au voisinage de $E^{-1}(0)$ et définissant $E^{-1}(\mathcal{F})$. Toujours par Lévi, il existe $\eta$ méromorphe à l'origine de $\mathbb{C}^{3}$ telle que $E^{*} \eta=\tilde{\eta}$. Cet $\eta$ convient.

Avant de s'intéresser à des centres dégénérés mentionnons que l'énoncé 8 ne se généralise pas en degré plus grand que deux. En voici la raison; considérons un feuilletage $\mathcal{F}^{\prime}$ de degré $\geq 2$ en $\mathbb{P}_{\mathbb{C}}^{2}$. Si $\mathcal{F}^{\prime}$ est générique il ne possède pas de courbe algébrique invariante $([16,9])$ et par suite il ne peut être défini par une 1 -forme fermée. Choisissons une carte affine $\mathbb{C}^{2}=\left\{\left(x_{1}, x_{2}\right)\right\} \subset \mathbb{P}_{\mathbb{C}}^{2}$ telle que l'origine $(0,0)$ soit un point non singulier de $\mathcal{F}^{\prime}$ et telle que la feuille passant par $(0,0)$ soit tangente à l'axe $x_{2}=0$ en $(0,0)$; en d'autre termes $\mathcal{F}^{\prime}$ est défini à l'origine par une 1 -forme du type $d x_{2}+\cdots$. Considérons l'application birationelle définie par $\sigma\left(x_{1}, x_{2}\right)=\left(x_{2}, x_{1} x_{2}\right)$; le feuilletage $\sigma^{-1}\left(\mathcal{F}^{\prime}\right)=\mathcal{F}$ a une intégrale première de Morse en $(0,0)$, c'est donc un centre, mais n'est pas défini par une 1 -forme fermée rationnelle puisque $\mathcal{F}^{\prime}$ ne l'est pas.

\section{Points nilpotents GÉNÉRiques}

Nous nous proposons de préciser le résultat du Théorème 10 en établissant d'abord un théorème de type Dulac dans le cas nilpotent générique. Une démonstration de ce même résultat a été proposée par C. Rousseau et C. Christopher suite à une discussion avec l'un des auteurs (communication personnelle).

Théorème 11. Soit $\mathcal{F}$ un feuilletage de degré deux sur le plan projectif $\mathbb{P}_{\mathbb{C}}^{2}$. On suppose que $\mathcal{F}$ possède un point singulier $m$ nilpotent tel que $\mu(\mathcal{F}, m)=2$. Si $\mathcal{F}_{, m}$ possède une intégrale première holomorphe non constante, alors $\mathcal{F}$ est défini par une 1 -forme fermée rationelle.

Démonstration. Nous choisissons une carte affine $\mathbb{C}^{2}=\left\{\left(x_{1}, x_{2}\right)\right\} \subset \mathbb{P}_{\mathbb{C}}^{2}$ dont l'origine est le point $m$. Le feuilletage $\mathcal{F}$ est donc défini par une 1 -forme du type :

$$
\omega=2 x_{1} d x_{1}+A_{2} d x_{1}+B_{2} d x_{2}+q\left(x_{1} d x_{2}-x_{2} d x_{1}\right)
$$

où les $A_{2}, B_{2}$ et $q$ sont des polynômes de degré 2 . 
Comme le germe $\mathcal{F}_{, 0}$ a une intégrale première holomorphe non triviale, c'est une courbe généralisée au sens de [2]. En particulier si $f \in \mathcal{O}\left(\mathbb{C}^{2}, 0\right)$ est une intégrale première minimale de $\mathcal{F}_{, 0}([24])$, alors la multiplicité algébrique de $f$ est 2 et son nombre de Milnor $\mu(f ; 0)=2$ aussi. En résulte que $f$ est holomorphiquement conjugué à $x_{1}^{2}-x_{2}^{3}$. Ceci nous permet de supposer, à automorphisme de $\mathbb{P}_{\mathbb{C}}^{2}$ fixant 0 près, que

$$
f=x_{1}^{2}-x_{2}^{3}-\sum_{i \geq 4} f_{i}=\sum_{i \geq 2} f_{i}
$$

où les $f_{i}$ sont des polynômes homogènes de degré $i$; on note

$$
f_{k}=\sum_{i+j=k} a_{i j} x_{1}^{i} x_{2}^{j}
$$

Remarquons que l'on peut, quitte à changer $f$ en $l(f), l \in \operatorname{Diff}(\mathbb{C}, 0)$, supposer que $a_{2 i, 0}=0$ pour tout $i \geq 2$.

Le but est maintenant d'obtenir des renseignements sur les polynômes $A_{2}, B_{2}$ en exploitant formellement l'égalité

$$
0=\omega \wedge d f=\left(2 x_{1}+A_{2}-q x_{2}\right)\left(-3 x_{2}^{2}+\frac{\partial f_{4}}{\partial x_{2}}+\cdots\right)-\left(B_{2}+q x_{1}\right)\left(2 x_{1}+\frac{\partial f_{4}}{\partial x_{1}}+\cdots\right) .
$$

Visiblement on a $B_{2}=-3 x_{2}^{2}$; en considérant les termes de degré 4 dans (2) on constate que :

$$
2 x_{1} \frac{\partial f_{4}}{\partial x_{2}}-3 x_{2}^{2} A_{2}-2 x_{1}^{2} q=0
$$

et $A_{2}$ est divisible par $x_{1}$ :

$$
A_{2}=x_{1}\left(a x_{1}+b x_{2}\right), \quad a, b \in \mathbb{C} .
$$

Si l'on pose $q=P x_{1}^{2}+Q x_{1} x_{2}+R x_{2}^{2}$ on obtient (en utilisant la remarque $a_{2 i, 0}=0$ ) :

$$
f_{4}=\frac{3}{8} b x_{2}^{4}+\left(\frac{a}{2}+\frac{R}{3}\right) x_{1} x_{2}^{3}+\frac{Q}{2} x_{1}^{2} x_{2}^{2}+P x_{1}^{3} x_{2} .
$$

L'étude des termes d'ordre 5 dans (2) donne $R=-\frac{3 a}{8}$ et

$$
f_{5}=\left(-\frac{3 b^{2}}{20}-\frac{3 Q}{5}\right) x_{2}^{5}-\left(\frac{21}{64} a b+\frac{3 P}{2}\right) x_{2}^{4} x_{1}-\left(\frac{3 a^{2}}{16}+\frac{Q b}{6}\right) x_{2}^{3} x_{1}^{2}-\frac{(Q a+P b)}{4} x_{2}^{2} x_{1}^{3}-\frac{P a}{2} x_{1}^{4} x_{2}+a_{50} x_{1}^{5} .
$$

On fait ensuite $x_{2}=0$ dans les termes d'ordre 6 de (2) et l'on obtient $P=-\frac{3}{32} a b$. Finalement on a

$$
A_{2}=x_{1}\left(a x_{1}+b x_{2}\right) ; \quad B_{2}=-3 x_{2}^{2} ; \quad q=-\frac{3}{32} a b x_{1}^{2}+Q x_{1} x_{2}-\frac{3 a}{8} x_{2}^{2}
$$

et nous allons distinguer deux cas suivant que $a$ est nul ou non.

Cas $a=0$. La 1 -forme $\omega$ s'écrit donc:

$$
\omega=x_{1}\left(2+b x_{2}-Q x_{2}^{2}\right) d x_{1}+\left(Q x_{1}^{2} x_{2}-3 x_{2}^{2}\right) d x_{2}=\left(1+\frac{b}{2} x_{2}-\frac{Q}{2} x_{2}^{2}\right) d x_{1}^{2}+\left(Q x_{1}^{2} x_{2}-3 x_{2}^{2}\right) d x_{2}
$$

et apparait comme image réciproque de

$$
\omega^{\prime}=\left(1+\frac{b}{2} y-\frac{Q}{2} y^{2}\right) d x+\left(Q x y-3 y^{2}\right) d y
$$

par l'application $\sigma:\left(x_{1}, x_{2}\right) \rightarrow\left(x_{1}^{2}, x_{2}\right)$. Remarquons que toute forme de type $\omega$ ' précédente, puisque no singulière possède une intégrale première holomorphe et donc $\omega$ aussi. Ainsi l'existence d'une intégrale première ne donne pas plus d'information sur les coefficients $b$ et $Q$.

Un calcul direct montre que la conique d'équation $\mathcal{C}=b Q x-3 Q y^{2}+6=0$ est invariante par $\omega^{\prime}$. Par suite $\mathcal{C} \circ \sigma=0$ est invariante par $\omega$. On vérifie que $\omega^{\prime} /\left(1+\frac{b}{2} y-\frac{Q}{2} y^{2}\right) \mathcal{C}$ est fermée et par suite que $\omega /\left(1+\frac{b}{2} x_{2}-\frac{Q}{2} x_{2}^{2}\right)\left(b Q x_{1}^{2}-3 Q x_{2}^{2}+6\right)$ aussi ; ceci démontre le théorème lorsque $a=0$. 
Cas $a \neq 0$. On peut supposer $a=1$, à isomorphisme près. Donc

$$
\begin{gathered}
\omega=\left[2 x_{1}+x_{1}\left(x_{1}+b x_{2}\right)-x_{2}\left(-\frac{3}{32} b x_{1}^{2}+Q x_{1} x_{2}-\frac{3}{8} x_{2}^{2}\right)\right] d x_{1} \\
+\left[-3 x_{2}^{2}+x_{1}\left(-\frac{3}{32} b x_{1}^{2}+Q x_{1} x_{2}-\frac{3}{8} x_{2}^{2}\right)\right] d x_{2} .
\end{gathered}
$$

Il faut ici calculer explicitement les coefficients $a_{05}$ et $a_{15}$, ce qui se fait sans difficulté à partie de (2) :

$$
a_{15}=\frac{147}{1280} b^{2}+\frac{3 Q}{5}, \quad a_{15}+\frac{5}{8} a_{05}=0
$$

pour en déduire que $Q=\frac{3}{32} b^{2}$.

On constate alors que la droite $l=x_{1}+b x_{2}+8=0$ est invariante par $\omega$. Un calcul direct montre que $8 \omega=l d G-3 G d l$ où $G=x_{1}^{2}-x_{2}^{3}+\frac{3}{8} b x_{1}^{2} x_{2}+\frac{3}{8} x_{1}^{3} ;$ ainsi $\frac{\omega}{l G}$ est fermée, ce qui prouve le théorème dans ce second cas.

Le Théorème [1], combiné à la Proposition [6, nous permet d'améliorer le Théorème 10] comme il suit. Soit $\left[\mathcal{F}_{\omega_{3}}\right]$ un feuilletage de degré 2 sur $\mathbb{P}_{\mathbb{C}}^{2} ;$ nous dirons que $\left[\mathcal{F}_{\omega_{3}}\right]$ satisfait la propriété $\mathcal{P}$ lorsque :

$\mathcal{P}_{1}$ : Pour tout point singulier $m \in\left[\mathcal{F}_{\omega_{3}}\right]$, si $\theta$ est une 1 -forme définissant $\left[\mathcal{F}_{\omega_{3}}\right]_{, m}$ alors le $1-$ jet de $\theta$ en $m$ est non nul.

$\mathcal{P}_{2}$ : Si $j^{1} \theta_{, m}$ est nilpotent, i.e. conjugué à $x_{1} d x_{1}$, alors $\mu\left(\left[\mathcal{F}_{\omega_{3}}\right] ; m\right)=2$.

Remarque 13. La condition $\mathcal{P}$ est satisfaite pour un ouvert de Zariski de feuilletages de degré deux.

Remarque 14. Si $\mathcal{P}$ n'est pas satisfaite, alors ou bien il existe un point $m$ tel que, avec les notations précédentes, $j^{1} \theta_{, m}=0$, ou bien il y a un point nilpotent $m$ en lequel $\mu\left(\left[\mathcal{F}_{\omega_{3}}\right] ; m\right) \geq 3$. Nous étudions ces deux situations plus loin.

Théorème 12. Soit $\omega=\omega_{3}+\cdots \in \Omega^{1}\left(\mathbb{C}^{3}, 0\right)$ un germe de 1 -forme intégrable dont la partie homogène $\omega_{3}$ est dicritique et Cod Sing $\omega_{3} \geq 2$. Si $\left[\mathcal{F}_{\omega_{3}}\right]$ satisfait la propriété $\mathcal{P}$ on est dans l'une des deux situations suivantes (non exclusives):

- $\mathcal{F}_{\omega}$ est défini par une 1-forme fermée méromorphe.

- $\mathcal{F}_{\omega}$ est holomorphiquement conjugué à $\mathcal{F}_{\omega_{3}}$.

Démonstration. Si $\left[\mathcal{F}_{\omega_{3}}\right]$ est sans nilpotence on invoque le Théorème 10 Sinon $\left[\mathcal{F}_{\omega_{3}}\right]$ a un point singulier $m$ nilpotent non trivial en lequel $\mu\left(\left[\mathcal{F}_{\omega_{3}}\right] ; m\right)=2$. Si $\mathcal{F}_{\omega}$ n'est pas conjugué à $\mathcal{F}_{\omega_{3}}$ c'est qu'on ne peut appliquer le Lemme 2 en un certain point singulier (pour conclure comme dans la Proposition 3) ; si c'est en $m$, d'après les Propositions [5] et 6 le germe $\left[\mathcal{F}_{\omega_{3}}\right], m$ possède une intégrale première holomorphe non constante. D'après le Théorème 11$]\left[\mathcal{F}_{\omega_{3}}\right]$ est défini par une 1 -forme fermée rationnelle $\underline{\eta}$. Si c'est en un point $m^{\prime}$ où le 1 -jet est non nilpotent, alors $m^{\prime}$ est central et $\left[\mathcal{F}_{\omega_{3}}\right]$ est encore défini par une 1-forme fermée.

Théorème 13. Soit $\omega=\omega_{3}+\cdots \in \Omega^{1}\left(\mathbb{C}^{3}, 0\right)$ un germe de 1 -forme intégrable dont la partie homogène $\omega_{3}$ est dicritique et $\operatorname{Cod} \operatorname{Sing} \omega_{3} \geq 2$. S'il existe un point $m \in \operatorname{Sing}\left[\mathcal{F}_{\omega_{3}}\right]$ tel que $j^{1} \theta_{, m} \equiv 0$ ( $\theta$ définissant $\left[\mathcal{F}_{\omega_{3}}\right]$ en $m$ ) alors $\mathcal{F}_{\omega}$ est transversalement affine, i.e. il existe une 1 -forme méromorphe fermée $\omega_{1}$ telle que $d \omega=\omega \wedge \omega_{1}$.

Remarque 15. Il y a une généralisation du Théorème 13 en tout ordre, i.e. en remplaçant $\omega_{3}$ par $\omega_{\nu}$ et la condition $j^{1} \theta_{, m} \equiv 0$ par $j^{\nu-2} \theta_{, m} \equiv 0$. La conclusion et la preuve sont les mêmes.

Remarque 16. D'un point de vue formel, la fonction multivaluée $F=\exp -\int \omega_{1}$ est un facteur intégrant de $\omega: d\left(\frac{\omega}{f}\right)=0$. 
Démonstration du Théorème 13 . On se place dans la carte affine $x_{3}=1$ et l'on supose que le 1 -jet de $\zeta=\left.\omega_{3}\right|_{x_{3}=1}$ est nul au point $(0: 0: 1)$, i.e. :

$$
\zeta=\zeta_{2}+q\left(x_{1} d x_{2}-x_{2} d x_{1}\right)
$$

où $\zeta_{2}$ est une 1 -forme à coefficients homogènes de degré 2 non colinéaire à $x_{1} d x_{2}-x_{2} d x_{1}$. Un calcul élémentaire (par éclatement par exemple) montre qu'il existe une 1 -forme méromorphe fermée $\underline{\eta}$ telle que $d \zeta=\zeta \wedge \underline{\eta}$. Comme dans la preuve du Théorème 10, en utilisant le Théorème de Frobenius et le Théorème d'Extension de Lévi, on construit une 1-forme méromorphe fermée $\tilde{\eta}$ définie au voisinage de $E^{-1}(0)$ telle que $d E^{*} \omega=E^{*} \omega \wedge \tilde{\eta}$. Toujours d'après Lévi il existe une 1 -forme méromorphe fermée $\omega$ telle que $E^{*} \omega_{1}=\tilde{\eta}$. Cet $\omega_{1}$ convient.

Conjecture. Soit $\left[\mathcal{F}_{\omega_{3}}\right]$ un feuilletage de degré deux sur $\mathbb{P}_{\mathbb{C}}^{2}$. Alors si $\omega=\omega_{3}+\cdots \in \Omega^{1}\left(\mathbb{C}^{3}, 0\right)$ est intégrable on a l'alternative :

(1) $\mathcal{F}_{\omega}$ et $\mathcal{F}_{\omega_{3}}$ sont conjugués holomorphiquement.

(2) $\mathcal{F}$ est défini par une 1 -forme fermée ou bien est transversalement affine.

Dans les paragraphes qui suivent on traite plusieurs nouveaux cas allant dans le sens de la Conjecture.

\section{Vers la CONJECTURE : LE CAS $\mu=3$}

Nous gardons la notation du paragraphe précédent et nous proposons quelques résultats en présence d'un point nilpotent $m$ tel que $\mu\left(\left[\mathcal{F}_{\omega_{3}}\right] ; m\right) \geq 3$. Comme on l'a dit précédemment un feuilletage de degré deux a au plus 7 points singuliers comptés avec multiplicité, i.e. $\mu\left(\left[\mathcal{F}_{\left.\omega_{3}\right]}\right] ; m\right) \leq 7$ et lorsque l'on classifie, parmi ces feuilletages, ceux qui ne présentent qu'un point singulier (i.e. $\mu\left(\left[\mathcal{F}_{\omega_{3}}\right] ; m\right)=7$ ) on constate que le 1 -jet de $\left[\mathcal{F}_{\omega_{3}}\right]_{, m}$ est non nilpotent $[8]$; de sorte que si $m$ est une singularité nilpotente de $\left[\mathcal{F}_{\omega_{3}}\right]$ on a $\mu\left(\left[\mathcal{F}_{\omega_{3}}\right] ; m\right) \in\{2, \ldots, 6\}$.

En vertu du Théorème 7 nous allons nous intéresser au feuilletages de degré deux $\left[\mathcal{F}_{\omega_{3}}\right]$ possédant un point singulier nilpotent $m$ en lequel $\left[\mathcal{F}_{\omega_{3}}\right]_{, m}$ possède un facteur intégrant formel. Pour celà nous avons besoin de résultats préparatoires élémentaires.

Soit $\mathcal{F}$ un feuilletage de degré 2 donné dans la carte affine $\left\{\left(x_{1}, x_{2}\right)\right\}=\mathbb{C}^{2} \subset \mathbb{P}_{\mathbb{C}}^{2}$ par la 1 -forme polynomiale :

$$
\theta=\left(x_{1}+A_{2}-q x_{2}\right) d x_{1}+\left(B_{2}+q x_{1}\right) d x_{2}
$$

où les $A_{2}, B_{2}$ et $q$ sont des polynômes homogènes de degré 2 .

Lemme 3. Si $\mu(\mathcal{F} ; 0) \geq 3$ alors la droite $x_{1}=0$ est invariante.

Démonstration. Si ce n'est pas le cas alors $B_{2}\left(0, x_{2}\right)$ est non nul et $\mu(\mathcal{F} ; 0)=2$.

Nous allons adopter les notations suivantes:

$$
A_{2}=\alpha x_{1}^{2}+\beta x_{1} x_{2}+\gamma x_{2}^{2}, \quad B_{2}=x_{1}\left(a x_{1}+b x_{2}\right), \quad q=P x_{1}^{2}+Q x_{1} x_{2}+R x_{2}^{2} .
$$

En restriction à $x_{1}=0$ on a $\theta=\left(\gamma x_{2}^{2}-R x_{2}^{3}\right) d x_{1}$; notons que $\gamma$ et $R$ ne peuvent être simultanément nuls. Si $\gamma$ est non nul, $\mathcal{F}$ a deux singularités distinctes sur la droite $x_{1}=0$ (et peut-être d'autres

ailleurs); dans ce cas on peut supposer, à automorphisme de $\mathbb{P}_{\mathbb{C}}^{2}$ près, que $\gamma=1$ et $R=0$. Après cette normalisation les singularités de $\mathcal{F}$ sur $x_{1}=0$ sont en 0 et à l'infini. À l'inverse si $\gamma$ est nul, $\mathcal{F}$ a une seule singularité sur $x_{1}=0$ qui est bien sûr le point 0 . Dans ce cas on peut supposer que $R=1$.

Nous résumons ces faits dans le

Lemme 4. Si $\mu(\mathcal{F} ; 0) \geq 3$ alors à automorphisme de $\mathbb{P}_{\mathbb{C}}^{2}$ près $\theta$ appartient à l'une des deux familles :

$$
\begin{gathered}
\Omega_{1}:=\left\{\theta_{1}=x_{1}\left[\left(1+\alpha x_{1}+\beta x_{2}\right) d x_{1}+\left(a x_{1}+b x_{2}\right) d x_{2}\right]+\left(P x_{1}^{2}+Q x_{1} x_{2}+x_{2}^{2}\right)\left(x_{1} d x_{2}-x_{2} d x_{1}\right)\right\} \\
\Omega_{2}:=\left\{\theta_{2}=x_{1}\left[\left(1+\alpha x_{1}+\beta x_{2}\right) d x_{1}+\left(a x_{1}+b x_{2}\right) d x_{2}\right]+x_{2}^{2} d x_{1}+\left(P x_{1}^{2}+Q x_{1} x_{2}\right)\left(x_{1} d x_{2}-x_{2} d x_{1}\right)\right\} .
\end{gathered}
$$


Remarque 17. On note que pour la première famille $\Omega_{1}$ on a $\mu\left(\mathcal{F}_{\theta_{1}} ; 0\right) \geq 4$. Dans la seconde famille $\Omega_{2}$ on a $\mu\left(\mathcal{F}_{\theta_{2}} ; 0\right)=3$ si et seulement si le coefficient $b$ est non nul. Si $b=0$ et $a \neq 0$ on a $\mu\left(\mathcal{F}_{\theta_{2}} ; 0\right)=4$; si $a=b=0$ et $Q \neq 0$ alors $\mu\left(\mathcal{F}_{\theta_{2}} ; 0\right)=5$ et enfin si $a=b=Q=0$ alors $\mu\left(\mathcal{F}_{\theta_{2}} ; 0\right)=6$.

Exemples. La 1 -forme $\theta_{1}=\left(x_{1}-x_{2}^{3}\right) d x_{1}+x_{1} x_{2}^{2} d x_{2}$ fait partie de la première famille $\Omega_{1}$; on a $\mu\left(\mathcal{F}_{\theta_{1}} ; 0\right)=5$ et $\mathcal{F}_{\theta_{1}}$ possède l'intégrale première rationnelle $\frac{1}{2 x_{1}^{2}}-\frac{1}{3} \frac{x_{2}^{3}}{x_{1}^{3}}$. Dans la seconde famille $\Omega_{2}$ on trouve par exemple (à homothétie près) $\theta_{2}=\left(x_{1}+x_{2}^{2}\right) d x_{1}+x_{1} d\left(x_{1}+x_{2}^{2}\right)=\left(2 x_{1}+x_{2}^{2}\right) d x_{1}+2 x_{1} x_{2} d x_{2}$ qui est fermée avec intégrale première $x_{1}\left(x_{1}+x_{2}^{2}\right)$. Ici on a $\mu\left(\mathcal{F}_{\theta_{2}} ; 0\right)=3$ et $b=2$.

Génériquement sur les paramètres $(\alpha, \beta, a, b, P, Q)$ un feuilletage $\mathcal{F}_{\theta_{2}}$ induit par un élément $\theta_{2} \in \Omega_{2}$ possède à l'origine deux germes de courbes invariantes distinctes : l'une est l'axe $x_{1}=0$ et l'autre est lisse et tangente à $x_{1}=0$. Pour des valeurs spéciales des paramètres ceci n'est pas toujours le cas. Pour voir ceci voici comment l'on procède; le germe linéaire $\left(x_{1}+x_{2}\right) d x_{1}-x_{1} d x_{2}$ possède un seul germe de courbe invariante à l'origine, l'axe $x_{1}=0$. Son image réciproque $\theta_{3}$ par le revêtement ramifié $\left(x_{1}, x_{2}^{2}\right)$ :

$$
\theta_{3}=\left(x_{1}+x_{2}^{2}\right) d x_{1}-2 x_{1} x_{2} d x_{2}
$$

possède la même propriété. La 1 -forme $\theta_{3}$ est un élément de $\Omega_{2}$, avec $b=-2$ et tous les autres paramètres nuls. Le feuilletage $\mathcal{F}_{\theta_{3}}$ est défini par la 1 -forme fermée $\frac{\theta_{3}}{x_{1}^{3}}$, ce qui fait apparaître le facteur intégrant $x_{1}^{3}$ qui possède donc de la multiplicité. Ce facteur intégrant est unique à constante multiplicative près.

Soit $\chi \subset \mathbb{Q}$ l'ensemble :

$$
\chi:=\left\{\frac{l-2}{k-1}, l \in \mathbb{N}, k \in \mathbb{N}-\{1\}\right\}=\mathbb{Q}>0 \cup\{-\mathbb{N}\} \cup\left\{\frac{-1}{\mathbb{N}}\right\} \cup\left\{\frac{-2}{\mathbb{N}}\right\}
$$

avec des notations évidentes. À l'inverse de cet exemple on a sous une hypothèse de non résonance le lemme technique suivant :

Lemme 5. Soit $\theta_{2}$ un élément de $\Omega_{2}$. On suppose que $\theta_{2}$ possède un facteur intégrant formel $f \in \widehat{\mathcal{O}\left(\mathbb{C}^{2}, 0\right)}: d\left(\frac{\theta_{2}}{f}\right)=0$. Si $b \notin \chi$, alors à constante multiplicative non nulle près $f=x_{1} g$ où $\left.g \in \widehat{\mathcal{O}\left(\mathbb{C}^{2}, 0\right.}\right)$ est une submersion formelle satisfaisant $\frac{\partial g}{\partial x_{1}}(0) \neq 0$ et $g\left(0, x_{2}\right)=x_{2}^{2}$.

Démonstration. La condition $d\left(\frac{\theta_{2}}{f}\right)=0$ s'exprime comme suit :

$$
f d \theta_{2}+\theta_{2} \wedge d f=0
$$

Remarquons que si $f$ est constant alors $\theta_{2}$ est fermée; ceci implique que $b=2$, ce qui est interdit par hypothèse. On écrit alors $f=x_{1}^{k} g\left(x_{1}, x_{2}\right)$ avec $k \in \mathbb{N}$ et $g \in \widehat{\mathcal{O}\left(\mathbb{C}^{2}, 0\right)}$ une série formelle telle que $g\left(0, x_{2}\right) \not \equiv 0$.

En dévelopant (3) et en faisant $x_{1}=0$ après simplification par $x_{1}^{k}$ on obtient :

$$
g\left(0, x_{2}\right)(b(1-k)-2)+x_{2} \frac{\partial g}{\partial x_{2}}\left(0, x_{2}\right) \equiv 0
$$

Quitte à multiplier $f$ par une constante, on peut supposer que

$$
g\left(0, x_{2}\right)=x_{2}^{l}+\cdots \quad \text { avec } l \in \mathbb{N} .
$$

On obtient alors d'après (44)

$$
b(1-k)-2+l=0
$$

qui conduit, puisque $b \notin \chi$, à $k=1$ et $l=2$.

Puisque maintenant $k=1$ la solution générale de (44) est $\left\{\lambda x_{2}^{2}, \lambda \in \mathbb{C}\right\}$, si bien que $g\left(0, x_{2}\right) \equiv x_{2}^{2}$. On écrit le développement en série de $g$ sous la forme suivante :

$$
g=x_{2}^{2}+x_{1} \varphi_{1}\left(x_{2}\right)+x_{1}^{2} \varphi_{2}\left(x_{2}\right)+\cdots+x_{1}^{i} \varphi_{i}\left(x_{2}\right)+\cdots
$$

où les $\varphi_{i}$ sont des séries formelles en $x_{2}$. Un calcul élémentaire montre que (3) implique :

$$
x_{2}^{2}\left((a-\beta)+5 Q x_{2}\right)+2 x_{2}\left(1+\beta x_{2}-Q x_{2}^{2}\right)+x_{2}^{2} \varphi_{1}^{\prime}\left(x_{2}\right)-(b+2) x_{2} \varphi_{1}\left(x_{2}\right)=0 .
$$


En particulier le calcul du coefficient de $x_{2}$ dans (5) conduit à

$$
2-(b+2) \varphi_{1}(0)=0
$$

et par suite $\varphi_{1}(0)=\frac{\partial g}{\partial x_{1}}(0)$ est non nul.

Remarque 18. Il y a une possibilité de preuve directe en utilisant que pour $b \notin \mathbb{Q}$ la réduction des singularités en 0 est celle de $\left(x_{1}+x_{2}^{2}\right) d x_{1}+b x_{1} x_{2} d x_{2}$.

Nous énonçons maintenant deux résultats voisins qui vont se substituer dans certains cas au Théorème de Dulac. Comme nous n'utiliserons pas explicitement le premier nous n'en donnons pas la preuve que nous avons vérifiée au prix d'un calcul long et lourd ne mettant en jeu que des arguments utilisés dans le Théorème 11 .

Théorème 14. Soit $\mathcal{F}$ un feuilletage de degré deux possédant une singularité nilpotente en 0 telle que $\mu(\mathcal{F} ; 0)=3$. Si $\mathcal{F}_{, 0}$ possède un facteur intégrant formel réduit du type $f=x_{1} g\left(x_{1}, x_{2}\right), g=x_{1}+\cdots$ alors $\mathcal{F}$ est défini par une 1 -forme fermée rationnelle.

Comme on l'a vu si $\mathcal{F}$ a une singularité nilpotente telle que $\mu(\mathcal{F} ; 0)=3$, alors $\mathcal{F}$ est décrit, à isomorphisme près, par une 1 -forme $\theta_{2} \in \Omega_{2}$ et le nombre $b=b(\mathcal{F})$ est bien défini. On a le :

Théorème 15. Soit $\mathcal{F}$ un feuilletage de degré deux possédant une singularité nilpotente en 0 telle que $\mu(\mathcal{F} ; 0)=3$. On suppose que $\mathcal{F}_{, 0}$ possède un facteur intégrant formel. Si $b=b(\mathcal{F}) \notin \mathbb{Q}$ alors $\mathcal{F}$ est défini par une 1-forme fermée rationnelle.

Le Lemme 5 fait que le Théorème 15 est un corollaire de Théorème 14.

Démonstration du Théorème 15 . Au point 0 le feuilletage $\mathcal{F}$ est défini par une 1 -forme de type $\theta_{2} \in \Omega_{2}$; d'après le Lemme [5 le facteur intégrant formel est de type $f=x_{1} g, g\left(0, x_{2}\right)=x_{2}^{2}, \frac{\partial g}{\partial x_{1}}(0) \neq 0$. Comme $\frac{\theta_{2}}{f}$ est fermée, il existe des nombres complexes $\lambda$ et $\mu$ et $V \in \widehat{\mathcal{O}\left(\mathbb{C}^{2}, 0\right)}$ tels que (voir [12] par exemple) :

$$
\frac{\theta_{2}}{f}=\frac{\theta_{2}}{x_{1} g}=\lambda \frac{d x_{1}}{x_{1}}+\mu \frac{d g}{g}+d V .
$$

Un calcul élémentaire (direct ou en utilisant la réduction des singularités de $\mathcal{F}_{, 0}$ ) montre que $\frac{\mu}{\lambda}=\frac{b}{2}$. En résulte que si $b$ est non réel positif ou un irrationnel mal approché par les rationnels (conditions de Siegel-Brujno), alors $f$ est holomorphe en 0 (voir [12] par exemple) : c'est une conséquence des théorèmes de Poincaré-Siegel appliqués en un point convenable de la réduction des singularités de $\mathcal{F}_{, 0}$.

$\mathrm{Au}$ point singulier à l'infini de $x_{1}=0$, le feuilletage est défini par une 1 -forme $\theta^{\prime}$ dont le 1 -jet est :

$$
j^{1} \theta^{\prime}=\left(x_{3}-Q x_{1}\right) d x_{1}-(b+1) x_{1} d x_{3} .
$$

Sous les mêmes conditions diophantiennes sur $b, \theta^{\prime}$ est holomorphiquement linéarisable et possède donc un facteur intégrant holomorphe.

Maintenant, on observe que le problème est invariant sous l'action d'automorphismes du corps $\mathbb{C}$ (existence d'un facteur intégrant formel, feuilletage défini par une forme fermée rationnelle); si le nombre $b$, qui est non rationnel, est algébrique il satisfait aux conditions diophantiennes exigées (c'est un résultat de Liouville). Si $b$ est transcendant on peut via l'action d'un automorphisme $\sigma$ se ramener au cas où encore ces conditions sont satisfaites.

Remarquons que l'holonomie de la variété invariante $x_{1}=0$ du feuilletage défini par $x_{3} d x_{1}-(b+$ 1) $x_{1} d x_{3}$ n'est pas d'ordre fini si $b \notin \mathbb{Q}$; il en est donc de même pour l'holonomie de la variété invariante $\left(\left(x_{1}=0\right)-\{0\}\right)$ pour $\mathcal{F}$. Considérons maintenant $\theta_{2}$ vue comme 1 -forme rationnelle globale sur $\mathbb{P}_{\mathbb{C}}^{2}$. On dispose en 0 d'un facteur intégrant holomorphe $f: d\left(\frac{\theta_{2}}{f}\right)=0$. Comme $\mathcal{F}$ n'a pas de singularités sur $\Sigma=\mathbb{C}^{2} \cap\left(\left(x_{1}=0\right)-\{0\}\right)$ qui est invariante on peut prolonger $f$ holomorphiquement le long de $\Sigma$. Au point à l'infini $\infty \in \bar{\Sigma}-\Sigma$, on dispose cette fois d'un facteur intégrant méromorphe $f_{\infty}$ hérité de la linéarisation de $\theta^{\prime}$. Si $\Delta$ est un petit disque transverse à $\Sigma$ au point $m_{0} \in \Sigma, m_{0}$ proche de $\infty$, les 1 -formes méromorphes fermées $\left.\frac{\theta_{2}}{f}\right|_{\Delta}$ et $\left.\frac{\theta_{2}}{f_{\infty}}\right|_{\Delta}$ sont invariantes par l'holonomie $h$ de la variété 
invariante $\Sigma$ réprésentée sur la transversale $\Delta$. Comme $h$ n'est pas d'ordre fini $\left.\frac{\theta_{2}}{f}\right|_{\Delta}$ et $\left.\frac{\theta_{2}}{f_{\infty}}\right|_{\Delta}$ sont $\mathbb{C}$-colinéaires. On peut donc, quitte à multiplier $f_{\infty}$ par une constante, supposer que :

$$
\left.\frac{\theta_{2}}{f}\right|_{\Delta}=\left.\frac{\theta_{2}}{f_{\infty}}\right|_{\Delta} .
$$

Par suite au voisinage de $x_{1}=0$ dans $\mathbb{P}_{\mathbb{C}}^{2}$ le feuilletage $\mathcal{F}$ est défini par une 1 -forme méromorphe fermée. Cette 1-forme s'étend en une 1-forme méromorphe globale sur $\mathbb{P}_{\mathbb{C}}^{2}$, qui est donc rationnelle.

Avec les notations précédentes on a le :

Corollaire 2. Soit $\omega=\omega_{3}+\cdots \in \Omega^{1}\left(\mathbb{C}^{3}, 0\right)$ un germe de 1 -forme intégrable à l'origine de $\mathbb{C}^{3}$ dont la partie homogène $\omega_{3}$ est dicritique et $\mathrm{Cod} \operatorname{Sing} \omega_{3} \geq 2$. On suppose que le feuilletage de degré deux $\left[\mathcal{F}_{\omega_{3}}\right]$ possède un point singulier $m \in \mathbb{P}_{\mathbb{C}}^{2}$ tel que $\mu\left(\left[\mathcal{F}_{\omega_{3}}\right] ; m\right)=3 ;$ si $b=b\left(\left[\mathcal{F}_{\omega_{3}}\right]\right)$ est non rationnel on a l'alternative :

(1) $\mathcal{F}_{\omega}$ est défini par une 1-forme fermée méromorphe.

(2) $\mathcal{F}_{\omega}$ est holomorphiquement conjugué à $\mathcal{F}_{\omega_{3}}$.

Démonstration. Commençons par quelques précisions. On peut supposer que $\left[\mathcal{F}_{\omega_{3}}\right]$ est donné en carte affine par :

$$
\theta_{2}=\left[x_{1}\left(1+\alpha x_{1}+\beta x_{2}\right)+x_{2}^{2}-x_{2}\left(P x_{1}^{2}+Q x_{1} x_{2}\right)\right] d x_{1}+x_{1}\left(a x_{1}+b x_{2}+P x_{1}^{2}+Q x_{1} x_{2}\right) d x_{2} .
$$

Au point singulier $m^{\prime}$ à l'infini de $x_{1}=0$, le feuilletage est donnée par $\theta^{\prime} \in \Omega^{1}\left(\mathbb{P}_{\mathbb{C}}^{2}, m^{\prime}\right)$, avec pour $1-$ jet $j^{1} \theta^{\prime}=\left(x_{3}-Q x_{1}\right) d x_{1}-(b+1) x_{1} d x_{3}$. En particulier $\mu\left(\left[\mathcal{F}_{\omega^{\prime}}\right] ; m^{\prime}\right)$ est plus grand ou égal que 1 (et même vaut 1 si $b$ est différent de -1 ). Par suite si $m^{\prime \prime}$ est un point singulier distinct de $m$ et $m^{\prime}$ alors $\mu\left(\left[\mathcal{F}_{\omega_{3}}\right] ; m^{\prime \prime}\right) \leq 3$. En résulte que si $\theta^{\prime \prime} \in \Omega^{1}\left(\mathbb{P}_{\mathbb{C}}^{2}, m^{\prime \prime}\right)$ est une 1 -forme définissant $\left[\mathcal{F}_{\omega_{3}}\right], m^{\prime \prime}$ alors le $1-$ jet $j^{1} \theta_{, m^{\prime \prime}}^{\prime \prime}$ est non identiquement nul. Supposons que $j^{1} \theta_{,{ }^{\prime \prime}}^{\prime \prime}$ soit nilpotent avec $\mu\left(\left[\mathcal{F}_{\omega_{3}}\right] ; m^{\prime \prime}\right)=3$.

Comme $m^{\prime \prime}$ ne peut être situé sur $x_{1}=0$ et $\left[\mathcal{F}_{\omega_{3}}\right]_{, m}$ ne possède qu'une droite invariante, le feuilletage $\left[\mathcal{F}_{\omega_{3}}\right]$ possède une seconde droite invariante $L$ passant par $m^{\prime \prime}$ et coupant $x_{1}=0$ nécessairement en $m^{\prime}$; c'est une conséquence du Lemme 3. Par suite $L$ est ou bien une droite $x_{1}=\varepsilon$ ou bien la droite à l'infini $x_{3}=0$. Un calcul élémentaire montre que, quitte à conjuguer par une transformation de type $\left(\frac{x_{1}}{x_{1}-\varepsilon}, i \varepsilon^{1 / 2} \frac{x_{2}}{x_{1}-\varepsilon}\right)$, on peut supposer que $L$ est la droite à l'infini $x_{3}=0$, cette transformation laissant invariant l'espace $\Omega_{2}$ et le coefficient $b$. Ainsi $\theta_{2}$ est du type :

$$
\theta_{2}=\left(x_{1}\left(1+\alpha x_{1}+\beta x_{2}\right)+x_{2}^{2}\right) d x_{1}+x_{1}\left(a x_{1}+b x_{2}\right) d x_{2} .
$$

Sous nos hypothèses $\mathcal{F}_{\theta_{2}}$ a pour singularités les trois points $m, m^{\prime}, m^{\prime \prime}$. Un second calcul élémentaire montre que $\mathcal{F}_{\theta_{2}, m^{\prime \prime}}$ a une singularité nilpotente à nombre de Milnor 3 si et seulement si $\alpha=\beta=a=0$. Mais dans ce cas $\mathcal{F}_{\theta_{2}}$ est donné par une forme fermée méromorphe; en résulte que le feuilletage $\mathcal{F}_{\omega}$ aussi.

Dans la suite on peut donc supposer que le point $m$ est le seul point nilpotent à nombre de Milnor 3 ; les autres points singuliers ont donc leur nombre de Milnor $\leq 2$. Si $\mathcal{F}_{\omega}$ n'est pas conjugué à $\mathcal{F}_{\omega_{3}}$ c'est que l'on ne peut appliquer le Lemme 2, En utilisant les Théorèmes 7, 8, 11, 15] et la Proposition [5] suivant la nature des points singuliers, on constate que $\left[\mathcal{F}_{\omega_{3}}\right]$ est donné par une 1 -forme fermée méromorphe, et donc $\mathcal{F}_{\omega}$ aussi.

\section{9. $\operatorname{LE} \operatorname{CAS} \mu=4$}

Soit $\mathcal{F}$ un feuilletage de degré 2 ayant une singularité nilpotente en 0 ; on suppose que $\mu(\mathcal{F} ; 0)=4$. Alors $\mathcal{F}$ appartient, à conjugaison près, à la famille $\Omega_{1}$, i.e. est donné par une 1 -forme de type $\theta_{1}$ avec $b \neq 0$, ou bien à la famille $\Omega_{2}$ avec $b=0$ et $a \neq 0$. Nous dirons suivant le cas que $\mathcal{F}$ est de type $\Omega_{1}$ ou $\Omega_{2}$. Si $\mathcal{F}$ est de type $\Omega_{2}$, nous dirons que $\mathcal{F}$ est non radial à l'infini si $Q=Q\left(\theta_{2}\right)=Q(\mathcal{F})$ est non nul.

Proposition 7. Soit $\mathcal{F}$ un feuilletage de degré deux sur $\mathbb{P}_{\mathcal{C}}^{2}$ de type $\Omega_{2} ;$ on suppose que $b(\mathcal{F})=Q(\mathcal{F})=$ 0 , i.e. $\mu(\mathcal{F} ; 0) \geq 4$ et $\mathcal{F}$ est radial à l'infini. Alors $\mathcal{F}$ est transversalement projectif. 
Démonstration. $\mathcal{F}$ est défini au point $(0: 1: 0)$ par une 1 -forme dont le 1 -jet est $x_{1} d x_{3}-x_{3} d x_{1}$; tout feuilletage de degré 2 ayant une singularité de type radial est transversalement projectif (équation de Ricatti).

Nous allons démontrer le résultat qui suit.

Théorème 16. Soit $\omega=\omega_{3}+\cdots \in \Omega^{1}\left(\mathbb{C}^{3}, 0\right)$ intégrable, $\omega_{3}$ dicritique, Cod Sing $\omega_{3} \geq 2$. On suppose que $\left[\mathcal{F}_{\omega_{3}}\right]$ possède une singularité nilpotente $m$ avec $\mu\left(\left[\mathcal{F}_{\omega_{3}}\right] ; m\right)=4$ de type $\Omega_{i}$. Alors $\mathcal{F}_{\omega}$ est conjugué $\grave{a} \mathcal{F}_{\omega_{3}}$.

Démonstration. Dans la suite on suppose $m=(0: 0: 1)$ et que $\left[\mathcal{F}_{\omega_{3}}\right]$ est donné par une forme de type $\theta_{i}$. Dans les deux cas la réduction des singularités du point nilpotent se fait en deux éclatements. On note $E x(1)$ le premier diviseur apparaissant au premier éclatement, $E x(2)$ le second; sur $E x(1)$ il y a un seul point singulier $p_{3}=E x(1) \cap E x(2)$. Pour les deux configurations $\Omega_{1}, \Omega_{2}$, le point $p_{3}$ est une singularité résonnante [23] avec 1 -jet de type $x d y+2 y d x,(y=0)=E x(1)_{, p_{3}},(x=0)=E x(2)_{, p_{3}}$. Comme sur Ex(1) il n'y a qu'une singularité, l'holonomie de la variété invariante locale $(y=0)$ est triviale et d'après Mattei-Moussu [24] il y a une intégrale première de type $x^{2} y$ en $p_{3}$. Par suite l'holonomie de la variété invariante locale $E x(2)_{, p_{3}}$ est une involution $x \rightarrow-x$. On note $p_{1} \in E x(2)$ le point singulier où aboutit le transformé strict de la droite invariante $x_{1}=0$ pour $\theta_{i}$. Enfin il y a un troisième point singulier, noté $p_{2}$.

Dans la situation où $\mathcal{F}$ est de type $\Omega_{2}$, le point singulier $p_{1}$ est résonnant, tandis que le point $p_{2}$ est de type noeud-col. En $p_{2}$ arrive une courbe invariante formelle transverse à $E x(2)$. À l'inverse dans le cas où $\mathcal{F}$ est de type $\Omega_{1}$, le point $p_{2}$ est résonnant tandis que le point $p_{1}$ est un noeud-col mal-orienté au sens où la variété faible invariante en $p_{1}$ est précisement la droite $x_{1}=0$.

Concernant la configuration dans $\mathbb{P}_{\mathbb{C}}^{2}$ des autres points singuliers, nous affirmons qu'il n'existe pas d'autre point singulier nilpotent à nombre de Milnor 3. En effet s'il existait un tel point $m^{\prime}$, il serait de type $\Omega_{2}$ (avec $\left.b \neq 0\right) ; m^{\prime}$ n'étant pas sur $x_{1}=0$, il y aurait une droite invariante passant par $m^{\prime}$ et il y aurait donc sur cette droite un autre point singulier $m^{\prime \prime}$ avec 1 -jet non nilpotent; donc $m^{\prime \prime}$ est distinct de $m$, ce qui nous donne au moins 8 points singuliers comptés avec multiplicité et est donc absurde.

Par suite les autres points singuliers ont leur nombre de Milnor inférieur ou égal a 2, en particulier en chacun des points singuliers le 1-jet est non nul. En chaque point singulier $m^{\prime}$ autre que $m$, on a $\mu\left(\left[\mathcal{F}_{\omega_{3}}\right] ; m^{\prime}\right) \leq 2 ;$ s'il n'existe pas de champ tangent à $E^{-1}\left(\mathcal{F}_{\omega}\right)$ en $m^{\prime}$ et transverse au diviseur $E^{-1}(0)_{, m}$ alors $\left[\mathcal{F}_{\omega_{3}}\right]$ et donc $\mathcal{F}_{\omega}$ est défini par une forme fermée méromorphe. Reste à étudier $E^{-1}\left(\mathcal{F}_{\omega}\right)$ au point $m=(0: 0: 1)$. S'il n'y a pas de champ $X_{, m}$ comme ci-dessus on sait que $\left[\mathcal{F}_{\omega_{3}}\right]_{, m}=\mathcal{F}_{\theta_{i}}$ possède un facteur intégrant formel $f: d\left(\frac{\theta_{2}}{f}\right)=0$.

Pour terminer la démonstration du théorème il nous suffit d'établir le

Lemme 6. Si $\mu\left(\mathcal{F}_{\theta_{i}} ; 0\right)=4$, avec $\theta_{i} \in \Omega_{i}$, alors $\theta_{i}$ n'a pas de facteur intégrant formel.

Démonstration. Si tel était le cas, l'holonomie du diviseur exceptionnel $\operatorname{Ex}(2)$ serait abélienne [12]. Mais pour chaque $\theta_{i}$ il y a un point $p_{i}$ en lequel la singularité est de type noeud-col avec nombre de Milnor 2. En résulte que l'holonomie de la variété invariante locale $\operatorname{Ex}(2)_{, p_{1}}$ est du type $\varphi: x \rightarrow x+c x^{2}+\cdots, c \neq 0$. Mais un tel $\varphi$ ne peut commuter à une involution $x \rightarrow-x$.

\section{LE CAS $\mu=5$}

Soit $\mathcal{F}$ un feuilletage de degré deux à singularité nilpotente à l'origine de $\mathbb{C}^{2}$ avec $\mu(\mathcal{F} ; 0)=5$. On peut supposer $\mathcal{F}$ donné par une 1 -forme $\theta$ appartenant à l'une des familles $\Omega_{1}, \Omega_{2}$. Si $\theta=\theta_{1} \in \Omega_{1}$ on vérifie que $b=0$, i.e. que

$$
\theta_{1}=\left[x_{1}\left(1+\alpha x_{1}+\beta x_{2}\right)-x_{2}\left(P x_{1}^{2}+Q x_{1} x_{2}+x_{2}^{2}\right)\right] d x_{1}+x_{1}\left(a x_{1}+P x_{1}^{2}+Q x_{1} x_{2}+x_{2}^{2}\right) d x_{2} .
$$

Remarquons que l'on peut faire $P$ ou $Q$ nuls.

Si $\theta=\theta_{2} \in \Omega_{2}$ on a $a=b=0$ et $Q \neq 0$, i.e. après normalisation

$$
\theta_{2}=\left[x_{1}\left(1+\alpha x_{1}+\beta x_{2}\right)+x_{2}^{2}-x_{2}\left(P x_{1}^{2}+x_{1} x_{2}\right)\right] d x_{1}+x_{1}^{2}\left(P x_{1}+x_{2}\right) d x_{2} .
$$


Sous les hypothèses précédentes on a la

Proposition 8. Si $\theta_{2}$ possède un facteur intégrant formel, alors $\mathcal{F}_{\theta_{2}}$ est défini par une 1-forme fermée rationnelle.

Démonstration. La singularité $(0: 1: 0)$ est de type $(x+y) d x-x d y$. En résulte que l'holonomie de la variété invariante $x_{1}=0$ est de la forme $y \rightarrow \frac{y}{1-y}$. Dans la résolution des singularités de $\mathcal{F}_{\theta_{2}, 0}$, la singularité résonnante $p_{1}$ est donc holomorphiquement normalisable [23], sans intégrale première holomorphe. En particulier en $p_{1}$ il y a un unique facteur intégrant formel, à constante multiplicative près, et celui-ci est en fait convergent. Par suite si $f$ est un facteur intégrant formel de $\theta_{2}$ en 0 , son relevé en $p_{1}$ est convergent; en résulte que $f$ converge. On prolonge alors la forme méromorphe fermée $\frac{\theta_{2}}{f}$ le long de $x_{1}=0$, comme on l'a fait en Théorème 15, Ceci est possible car l'holonomie de $x_{1}=0$ est non périodique.

De la même façon on a la :

Proposition 9. Si $\theta_{1}$ possède un facteur intégrant formel $f$ alors $\mathcal{F}_{\theta_{1}}$ est défini par une 1-forme fermée rationnelle.

Démonstration. Si l'on procède à trois éclatements successifs (grosso modo, on pose $x_{1}=s x_{2}^{3}$ ) on trouve un point singulier dont le 1 -jet est $6 u d v-v d u$. Les théorèmes de linéarisation et normalisation de Poincaré assurent qu'en un tel point tout facteur intégrant formel est en fait convergent. Ceci implique que $f$ est convergent; la 1 -forme fermée méromorphe $\frac{\theta_{1}}{f}$ locale se prolonge le long de $\overline{x_{1}=0} \subset \mathbb{P}_{\mathbb{C}}^{2}$ puis à tout $\mathbb{P}_{\mathbb{C}}^{2}$.

Considérons un déploiement du germe de feuilletage $\mathcal{F}_{\theta_{2}}$; il suit des Proposition 6 et Remarque 11 que si ce déploiement est non trivial et si $\theta_{2}$ n'a pas de facteur intégrant formel alors la forme normale de Loray de $\theta_{2}$ est de type :

$$
\theta_{L}=x d x+\left(y^{4}+x l\left(y^{2}\right)\right) d y^{2} \text { avec } \lambda=l(0)
$$

plus précisément il existe des coordonnées $x, y$ et une unité $U=1+u_{1} x+u_{2} y+\cdots$ telles que $\theta_{2}=U \theta_{L} ;$ on constate que $d \theta_{2}=\left(-\beta x_{1}-2 x_{2}+\cdots\right) d x_{1} \wedge d x_{2}$. En particulier la tangente aux zéros de $d \theta_{2}\left(2 x_{2}+\beta x_{1}=0\right)$ est différente du cône tangent $x_{1}=0$ de $\theta_{2}$. Maintenant le cône tangent de $\theta_{L}$ est $x=0$ et celui de $d \theta_{L}$ est $2 \lambda y-u_{2} x=0$. Pour qu'ils soient distincts il est nécessaire que $\lambda$ soit non nul. La Remarque 11] dit que, sous cette hypothèse, $\theta_{2}$ possède un facteur intégrant formel.

Remarque 19. La 1 -forme $\theta_{2}=\left(x_{1}+x_{2}^{2}-x_{1} x_{2}^{2}\right) d x_{1}+x_{1}^{2} x_{2} d x_{2}$ satisfait la Proposition 8 i.e. $\mathcal{F}_{\theta_{2}}$ est défini par une 1 -forme fermée rationnelle.

Examinons maintenant le cas où $\theta=\theta_{1} \in \Omega_{1}$; toujours sous l'hypothèse d'un déploiement non trivial la forme normale de Loray de $\theta_{1}$ est, si $\theta_{1}$ n'a pas de facteur intégrant, encore de la forme :

$$
\theta_{L}=x d x+\left(y^{4}+x l\left(y^{2}\right)\right) d y^{2} .
$$

On exploite ici le fait que l'involution $(x, y) \rightarrow(x,-y)$ laisse invariante $\theta_{L}$. Le feuilletage $\mathcal{F}_{\theta_{1}}$ est donc lui aussi laissé invariant par une involution $I$ de type $(x,-y)$. On considère alors le schéma de réduction des singularités de $\theta_{1}$; le premier éclatement produit un premier diviseur $\operatorname{Ex}(1)$ contenant un seul point singulier $p_{1}$ correspondant à $x_{1}=0$. On éclate alors $p_{1}$, ce qui produit un nouveau diviseur $E x(2)$ et deux points singuliers; le croisement $\operatorname{Ex}(1) \cap E x(2)$ noté encore $p_{1}$ et un point $p_{2} \neq p_{1}$ où aboutit $x_{1}=0$. En $p_{1}$ le feuilletage est réduit, mais il faut encore éclater $p_{2}$, ce qui produit un diviseur $E x(3)$ et trois points singuliers distincts : $p_{2}=E x(2) \cap E x(3), p_{3}$ où aboutit $x_{1}=0$ et un troisième point singulier $p_{4}$ distinct de $p_{2}$ et $p_{3}$. On relève l'involution $I$ à cette configuration pour obtenir une involution $\widetilde{I}$. La restriction de $\widetilde{I}$ à $E x(3)$ fixe les points $p_{2}$ et $p_{3}$. Un calcul élémentaire montre que $\left.\widetilde{I}\right|_{E x(3)}$ est de type $s \rightarrow-s$. Remarquons que puisque $I$ laisse invariante le feuilletage $\mathcal{F}_{\theta_{2}}$, le point $p_{4}$ doit être laissé fixe par $\widetilde{I}$; ce qui est absurde. Ce cas n'arrive donc pas. 
On en déduit en utilisant les mêmes idées que précédemment le :

Théorème 17. Soit $\omega=\omega_{3}+\cdots \in \Omega^{1}\left(\mathbb{C}^{3}, 0\right)$ intégrable, $\omega_{3}$ dicritique, Cod Sing $\omega_{3} \geq 2$. On suppose que $\left[\mathcal{F}_{\omega_{3}}\right]$ possède un point nilpotent $m$ tel que $\mu\left(\left[\mathcal{F}_{\omega_{3}}\right] ; m\right)=5$. Alors ou bien $\mathcal{F}_{\omega}$ et $\mathcal{F}_{\omega_{3}}$ sont conjuguées ou bien $\mathcal{F}_{\omega}$ est défini par une 1-forme fermée méromorphe.

\section{LE CAS $\mu=6$}

Soit $\mathcal{F}$ un feuilletage de degré deux sur $\mathbb{P}_{\mathbb{C}}^{2}$ ayant une singularité nilpotente $m=(0: 0: 1)$ avec $\mu(\mathcal{F} ; m)=6$. On peut supposer que $\mathcal{F}$ est défini par une 1 -forme $\theta$ appartenant à l'une des familles $\Omega_{i}$. Le lemme qui suit est élémentaire :

Lemme 7. Si $\mu(\mathcal{F} ; m)=6$ alors $\theta$ appartient à la famille $\Omega_{2}$; avec les notations habituelles on a de plus $a=b=Q=0$.

Ainsi $\mathcal{F}$ est défini par une 1 -forme de type

$$
\theta_{2}=\left[x_{1}\left(1+\alpha x_{1}+\beta x_{2}\right)+x_{2}^{2}-P x_{1}^{2} x_{2}\right] d x_{1}+P x_{1}^{3} d x_{2}
$$

avec $P \neq 0$. Quitte à faire un automorphisme de $\mathbb{P}_{\mathbb{C}}^{2} a d$-hoc, on se ramène à $P=1, \alpha=\beta=0$. On note $\theta_{2}^{0}$ la 1 -forme correspondant. Il y a donc un seul feuilletage de ce type à conjugaison près noté $\mathcal{F}_{0}$.

Proposition 10. Le feuilletage $\mathcal{F}_{0}$ est transversalement projectif.

Démonstration. Après éclatement du point à l'infini $(0: 1: 0), \mathcal{F}_{0}$ est transverse à la fibration $x_{1}=$ cte en dehors de la droite $x_{1}=0$, ce qui implique la proposition.

Le feuilletage $\mathcal{F}_{0}$ est défini dans la carte $x_{1}=1$ par

$$
d x_{2}-\left(x_{3}+x_{2}^{2}\right) d x_{3} .
$$

Il s'agit de la fameuse équation d'Airy utilisée pour les problèmes de diffraction. Liouville a démontré que les solutions de cette équation différentielle ne sont pas Liouvilliennes. Ceci signifie, en utilisant les travaux de Singer [26] et Casale [6] que $\mathcal{F}_{0}$ n'est pas transversalement affine : il n'existe pas de 1 -forme fermée rationnelle $\eta$ telle que $d \theta_{2}^{0}=\theta_{2}^{0} \wedge \eta$. En particulier $\mathcal{F}_{0}$ n'est pas défini par une 1 -forme fermée rationnelle.

En fait il y a une version locale de ce qui précède.

Lemme 8. La 1-forme $\theta_{2}^{0}$ ne possède pas de facteur intégrant formel.

Démonstration. Comme $Q=0$, la singularité $(0: 1: 0)$ de $\mathcal{F}_{0}$ est de type radial, i.e. possède une intégrale première de la forme $\frac{y}{x}=$ cte. En résulte que l'holonomie de la variété invariante $x_{1}=0$ est triviale. Dans la résolution des singularités ceci implique, d'après [24, qu'en $p_{1}$ le feuilletage transformé de $\mathcal{F}_{0}$ possède au point $p_{1}$ une intégrale première de type $u v^{2}=$ cte avec $(v=0)=\operatorname{Ex}(2)_{, p_{1}}$. Ceci implique aussi que, pour un bon choix de coordonnées, l'holonomie de la variété invariante $E x(2)$ au point $p_{1}$ est la symétrie $v \rightarrow-v$. On se place maintenant au point $p_{2}$ où le point singulier est de type noeud-col avec nombre de Milnor $4:$ au point $p_{2}$ le feuilletage est à conjugaison près du type

$$
(x+\cdots) d y+y^{4} d x
$$

où cette fois $(y=0)=E x(2)_{, p_{2}}$. Le difféomorphisme d'holonomie da la variété invariante $E x(2)_{, p_{2}}$ en $p_{2}$ est donc de la forme $y+c y^{4}+\cdots, c \in \mathbb{C}^{*}$. Notons qu'un tel difféomorphisme ne peut commuter avec une involution de type $y \rightarrow-y$. Or si $\theta_{2}^{0}$ possédait un facteur intégrant formel, le groupe d'holonomie du diviseur $E x(2)$ serait abélien. D'où le lemme.

On en déduit le

Théorème 18. Soit $\omega=\omega_{3}+\cdots \in \Omega^{1}\left(\mathbb{C}^{3}, 0\right)$ intégrable, $\omega_{3}$ dicritique, Cod Sing $\omega_{3} \geq 2$. On suppose que $\left[\mathcal{F}_{\omega_{3}}\right]$ possède une singularité nilpotente $m$ avec $\mu\left(\left[\mathcal{F}_{\omega_{3}}\right] ; m\right)=6$. Alors $\mathcal{F}_{\omega}$ est conjugué à $\mathcal{F}_{\omega_{3}}$.

Démonstration. C'est une conséquence du Lemme 8 . 


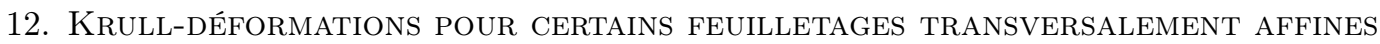

On s'intéresse ici aux feuilletages $\mathcal{F}_{\omega}$ où $\omega=\omega_{3}+\cdots \in \Omega^{1}\left(\mathbb{C}^{3}, 0\right)$ et $\left[\mathcal{F}_{\omega_{3}}\right]$ est transversalement affine comme dans le Théorème 13 On suppose que $\left[\mathcal{F}_{\omega_{3}}\right]$ est donné en carte affine par

$$
\theta_{0}=\omega_{2}+q\left(x_{1} d x_{2}-x_{2} d x_{1}\right)
$$

où $\omega_{2}$ est une 1 -forme à coefficients homogènes de degré deux et $q$ une forme quadratique. Pour un choix générique de $\omega_{2}$ et $q$, le feuilletage $\mathcal{F}_{\theta_{0}}$ n'est pas défini par une 1-forme fermée. En fait le feuilletage local $\mathcal{F}_{\theta_{0}}$ ne possède pas en général de facteur intégrant formel. Nous allons faire les hypothèses (génériques) supplémentaires suivantes :

(i) À conjugaison linéaire près $\omega_{2}=x_{1} x_{2}\left(x_{1}-x_{2}\right)\left(\lambda_{1} \frac{d x_{1}}{x_{1}}+\lambda_{2} \frac{d x_{2}}{x_{2}}+\lambda_{3} \frac{d\left(x_{2}-x_{1}\right)}{x_{2}-x_{1}}\right)$, $\lambda_{1}+\lambda_{2}+\lambda_{3}=1$, condition qui est réalisée dès que le cône tangent est réduit.

(ii) $\mu\left(\mathcal{F}_{\theta_{0}} ; 0\right)=4$, i.e. $\lambda_{1} \lambda_{2} \lambda_{3} \neq 0$.

(iii) Aux autres points singuliers $m_{i}$, que l'on peut supposer à distance finie, la condition de Kupka $d \theta\left(m_{i}\right) \neq 0$ est satisfaite.

(iv) Les $\lambda_{i}$ sont deux à deux distincts.

(v) $\mathcal{F}_{\theta_{0}}$ n'est pas défini par une 1-forme fermée.

Un calcul élémentaire montre que

$$
d \theta_{0}+\theta_{0} \wedge\left(\left(1+\lambda_{1}\right) \frac{d x_{1}}{x_{1}}+\left(1+\lambda_{2}\right) \frac{d x_{2}}{x_{2}}+\left(1+\lambda_{3}\right) \frac{d\left(x_{2}-x_{1}\right)}{x_{2}-x_{1}}\right)=0
$$

ce qui produit d'ailleurs une 1 -forme $\omega_{1}$ comme dans le Théorème 13 . Notons que puisque $\mathcal{F}_{\theta_{0}}$ n'est pas défini par une forme fermée, les $\lambda_{i}$ ne sont pas dans $\mathbb{Z}$.

Soit $\left[\mathcal{F}_{\omega_{3}}\right]$ défini par $\theta_{0}$ comme ci-dessus. Nous dirons que $\left[\mathcal{F}_{\omega_{3}}\right]$ est transversalement affine générique (TAG) si les conditions (i), (ii), (iii), (iv), (v) sont satisfaites.

Théorème 19. Soit $\mathcal{F}_{\omega}$ un germe de feuilletage à l'origine de $\mathbb{C}^{3}$ donné par $\omega=\omega_{3}+\cdots, \omega_{3}$ dicritique. Si $\left[\mathcal{F}_{\omega_{3}}\right]$ est TAG alors $\mathcal{F}_{\omega}$ et $\mathcal{F}_{\omega_{3}}$ sont conjugués.

Démonstration. Après un éclatement dans la carte où $E=\left(x_{1} x_{3}, x_{2} x_{3}, x_{3}\right)$ on a avec les notations précédentes :

$$
\theta=\frac{E^{*} \omega}{x_{3}^{4}}=\omega_{2}+q\left(x_{1} d x_{2}-x_{2} d x_{1}\right)+x_{3}\left(A d x_{1}+B d x_{2}\right)+h d x_{3} .
$$

Comme $\left.\theta\right|_{x_{3}=0}=\theta_{0}$, il existe une 1 -forme fermée méromorphe $\theta_{1}$ telle que $d \theta+\theta \wedge \theta_{1}=0$, avec

$$
\left.\theta_{1}\right|_{x_{3}=0}=\left(1+\lambda_{1}\right) \frac{d x_{1}}{x_{1}}+\left(1+\lambda_{2}\right) \frac{d x_{2}}{x_{2}}+\left(1+\lambda_{3}\right) \frac{d\left(x_{2}-x_{1}\right)}{x_{2}-x_{1}} .
$$

Puisque $\theta_{1}$ est construite par prolongement de $\left.\theta_{1}\right|_{x_{3}=0}, \theta_{1}$ n'a pas de pôle le long de $x_{3}=0$. La condition (iv) implique que $\theta_{1}$ a ses pôles le long de trois surfaces lisses deux à deux transverses dont les intersections avec $x_{3}=0$ sont $x_{1} x_{2}\left(x_{2}-x_{1}\right)=0$. À conjugaison holomorphe locale près on peut supposer que $\theta_{1}$ a ses pôles le long de :

$$
x_{1}=0, \quad x_{2}=0, \quad x_{2}-x_{1}+z^{k}=0
$$

pour un certain $k \in \mathbb{N}$. En résulte que la forme fermée $\theta_{1}$ est du type suivant en $(0,0,0)$ :

$$
\theta_{1}=\left(1+\lambda_{1}\right) \frac{d x_{1}}{x_{1}}+\left(1+\lambda_{2}\right) \frac{d x_{2}}{x_{2}}+\left(1+\lambda_{3}\right) \frac{d\left(x_{2}-x_{1}+z^{k}\right)}{x_{2}-x_{1}+z^{k}}+d f
$$

avec $f$ holomorphe en $(0,0,0), f\left(x_{1}, x_{2}, 0\right) \equiv 0$.

Soit $U$ une unité, $U(0)=1$, et $\phi$ le difféomorphisme :

$$
\phi=\left(x_{1} U^{k}, x_{2} U^{k}, x_{3} U\right) .
$$


On pose $\theta_{1}^{\prime}=\theta_{1}-d f$; un calcul direct montre que

$$
\phi^{*} \theta_{1}^{\prime}=\theta_{1}^{\prime}+4 k \frac{d U}{U} .
$$

Par suite, si $k$ est non nul, on peut supposer que $\theta_{1}^{\prime}=\theta^{\prime}$ (choisir $U=\exp \frac{-f}{4 k}$ ). Après cette transformation on a :

$$
d \theta+\theta \wedge\left(\left(1+\lambda_{1}\right) \frac{d x_{1}}{x_{1}}+\left(1+\lambda_{2}\right) \frac{d x_{2}}{x_{2}}+\left(1+\lambda_{3}\right) \frac{d\left(x_{2}-x_{1}+x_{3}^{k}\right)}{x_{2}-x_{1}+x_{3}^{k}}\right)=d \theta+\theta \wedge \theta_{1} .
$$

On considère alors l'application $\sigma$ :

$$
\sigma=\left(x_{1}, x_{2}, e^{\frac{2 \pi i}{k}} x_{3}\right) .
$$

On a $\sigma^{*} \theta_{1}=\theta_{1}$; on introduit la moyenne $\bar{\theta}$ de $\theta$ sous l'action de $\sigma$ :

$$
\bar{\theta}=\theta+\sigma^{*} \theta+\cdots+\sigma^{k-1 *} \theta
$$

qui est bien sûr invariante sous l'action de $\sigma$; on a $\left.\bar{\theta}\right|_{x_{3}=0}=k \theta_{0}$, ce qui indique que $\bar{\theta}$ est non triviale. L'invariance de $\bar{\theta}$ sous l'action de $\sigma$ dit que $\bar{\theta}$ est image réciproque par l'application $\varphi=\left(x_{1}, x_{2}, x_{3}^{k}\right)$ d'une certaine 1 -forme $\alpha$ vérifiant $\left.\alpha\right|_{x_{3}=0}=k \theta_{0}$ et :

$$
d \alpha+\alpha \wedge\left(\left(1+\lambda_{1}\right) \frac{d x_{1}}{x_{1}}+\left(1+\lambda_{2}\right) \frac{d x_{2}}{x_{2}}+\left(1+\lambda_{3}\right) \frac{d\left(x_{2}-x_{1}+x_{3}\right)}{x_{2}-x_{1}+x_{3}}\right)=d \alpha+\alpha \wedge \beta_{1} .
$$

On a donc un nouveau déploiement de $\theta_{0}$ mais avec $x_{1} x_{2}\left(x_{2}-x_{1}-x_{3}\right)=0$ comme hypersurface invariante.

Comme cette hypersurface est un croisement normal, lorsque l'on éclate l'origine par $E$, l'holonomie du diviseur exceptionnel $E^{-1}(0)$ pour $\mathcal{F}_{E^{*} \alpha}$ est abélienne. En résulte que l'holonomie du diviseur exceptionnel après un éclatement du feuilletage $\mathcal{F}_{\theta_{0}}$ est aussi abélienne. Ce qui est exclus par la condition (v). Par suite $k=0$ et $\theta$ possède la surface invariante $x_{1} x_{2}\left(x_{2}-x_{1}\right)=0$. Il est facile de voir que $\theta=F^{*} \theta_{0}$ où $F$ est une submersion $a d$-hoc. On conclut en invoquant le Lemme 8 ,

\section{Perturbations de 1-Formes fermées : exemples}

On considère dans ce paragraphe des feuilletages $\mathcal{F}_{\omega}, \omega=\omega_{3}+\cdots$ dont la partie de degré deux $\left[\mathcal{F}_{\omega_{3}}\right]$ est défini par une 1 -forme fermée rationnelle. Comme on l'a dit au paragraphe 6 , $\mathcal{F}_{\omega}$ est aussi défini par une 1 -forme fermée. Le cas le plus simple est celui où $\left[\mathcal{F}_{\omega_{3}}\right]$ est un pinceau générique de coniques. Un tel pinceau est à conjugaison linéaire près donné par les niveaux de $\frac{Q_{1}}{Q_{2}}=\frac{\left(x_{0}-x_{1}\right)\left(x_{0}+x_{1}\right)}{\left(x_{0}-x_{2}\right)\left(x_{0}+x_{2}\right)}$. Le feuilletage associé est donné en coordonnées homogènes par

$$
\omega_{3}=Q_{1} d Q_{2}-Q_{2} d Q_{1} .
$$

Vu dans $\mathbb{P}_{\mathbb{C}}^{2}$ le feuilletage $[\mathcal{F}]_{\omega_{3}}$ a sept singularités, dont quatre sont de type radial $((1: 1: 1)$, $(1: 1:-1),(1:-1: 1),(-1: 1: 1))$ et trois sont des centres $((1: 0: 0),(0: 1: 0),(0: 0: 1))$. Vu dans $\mathbb{C}^{3}$ le feuilletage $\mathcal{F}_{\omega_{3}}$ a donc sept lignes de singularités dont quatre sont de type Kupka $\left(Q_{1}=Q_{2}=0\right)$, et donc persistantes par perturbation. Soit $L=a_{0} x_{0}+a_{1} x_{1}+a_{2} x_{2}, a_{i} \in \mathbb{C}$, une forme linéaire; introduisons la 1 -forme intégrable $\omega_{L} \in \Omega^{1}\left(\mathbb{C}^{3}, 0\right)$

$$
\omega_{L}:=Q_{1} d Q_{2}-Q_{2} d Q_{1}+Q_{1} Q_{2} d L .
$$

On remarque de $\omega_{L}$ s'annule sur les quatre droites $Q_{1}=Q_{2}=0$ et que $\mathcal{F}_{\omega_{L}}$ a l'intégrale première méromorphe $e^{-L} \frac{Q_{1}}{Q_{2}}$. Considérons l'éclatement $E: \widetilde{\mathbb{C}}^{3} \rightarrow \mathbb{C}$ de l'origine; dans la première carte $\left(x_{0}, t_{1}, t_{2}\right)$ où $E=\left(x_{0}, t_{1} x_{0}, t_{2} x_{0}\right)$, on a :

$$
E^{*} \frac{\omega}{Q_{1} Q_{2}}=\frac{-2 t_{1} d t_{1}}{1-t_{1}^{2}}+\frac{2 d t_{2}}{1-t_{2}^{2}}+\left(a_{0}+a_{1} t_{1}+a_{2} t_{2}\right) d x_{0}+x_{0}\left(a_{1} d t_{1}+a_{2} d t_{2}\right) .
$$

En particulier si $a_{0} \neq 0$, la singularité initiale de $\left[\mathcal{F}_{\omega_{3}}\right]$ en $(1: 0: 0)$ a disparu et devient une tangence entre le feuilletage éclaté $E^{-1}\left(\mathcal{F}_{\omega_{L}}\right)$ et le diviseur exceptionnel $E^{-1}(0)$. Si $a_{0} a_{1} a_{2} \neq 0$, les singularités 
de $E^{-1}\left(\mathcal{F}_{\omega_{L}}\right)$ sont précisément sur les transformés strictes de $Q_{1}=Q_{2}=0$. Le long de ces quatre droites $D_{1}, D_{2}, D_{3}, D_{4}$ le feuilletage $E^{-1}\left(\mathcal{F}_{\omega_{L}}\right)$ est de type Kupka-radial. De sorte que si l'on éclate l'origine puis ces quatre droites par l'application $\sigma: M=\widetilde{\mathbb{C}}_{D_{1}, D_{2}, D_{3}, D_{4}}^{3} \rightarrow \mathbb{C}^{3}$, le feuilletage transformé $\sigma^{-1}(\mathcal{F})$ est maintenant sans singularités. On obtient ainsi en dimension trois un exemple de feuilletage absolument dicritique au sens de Cano-Corral [4]. On peut d'ailleurs généraliser cet exemple en prenant pour les $Q_{i}$ des polynômes de degré $k \geq 2$ génériques.

Remarquons qu'en annulant certains $a_{i}$ on peut obtenir pour $\mathcal{F}_{\omega_{L}}$ quatre, cinq, six ou sept droites singulières. Ainsi sur cet exemple on peut éliminer de façons indépendentes les droites singulières venant d'une configuration centrale.

En choisissant des entiers non nuls $k_{0}, k_{1}, k_{2}$ on peut aussi considérer les feuilletages donnés par $\omega=Q_{1} d Q_{2}-Q_{2} d Q_{1}+Q_{1} Q_{2} d f$ avec $f=a_{0} x_{0}^{k_{0}}+a_{1} x_{1}^{k_{1}}+a_{2} x_{2}^{k_{2}}$. Par exemple dans $E^{-1}\left(\mathbb{C}^{3}\right)=\widetilde{\mathbb{C}}^{3}$ le feuilletage relevé a au point $(1: 0: 0)$ une singularité avec intégrale première du type $x y+a_{0} z^{k_{1}}$. On en déduit une infinité non dénombrable de déformations de $\mathcal{F}_{\omega_{3}}$ non conjuguées.

On peut procéder de même dans le cas de formes logarithmiques de type $(1,1,1,1)$ suivant la notation de [9]. Un feuilletage générique de ce type est à conjugaison près de la forme :

$$
\omega_{3}=x_{0} x_{1} x_{2}\left(x_{0}+x_{1}+x_{2}\right)\left(\lambda_{0} \frac{d x_{0}}{x_{0}}+\lambda_{1} \frac{d x_{1}}{x_{1}}+\lambda_{2} \frac{d x_{2}}{x_{2}}-\frac{d\left(x_{0}+x_{1}+x_{2}\right)}{x_{0}+x_{2}+x_{2}}\right)
$$

avec $\lambda_{0}+\lambda_{1}+\lambda_{2}=1$. Les points singuliers dans $\mathbb{P}_{\mathbb{C}}^{2}$ sont au nombre de sept ; parmi eux six correspondent aux intersections des quatre droites invariantes. Ce sont des singularités logarithmiques génériques pour des valeurs génériques des paramètres $\lambda_{k}$. La septième singularité est de type centre et se trouve au point $\left(\lambda_{0}: \lambda_{1}: \lambda_{2}\right)$. Vu dans $\mathbb{C}^{3}$ le feuilletage $\mathcal{F}_{\omega_{3}}$ a donc sept droites singulières. On peut comme précédemment introduire

$$
\omega_{L}=\omega_{3}+x_{0} x_{1} x_{2}\left(x_{0}+x_{1}+x_{2}\right) d x_{0} .
$$

Le feuilletage $\mathcal{F}_{\omega_{L}}$ a seulement six droites singulières. On peut aussi considérer les 1 -formes $\omega_{3}+x_{0} x_{1} x_{2}\left(x_{0}+x_{1}+x_{2}\right) d f_{k}$ avec $f_{k}=x_{0}^{k}$ produisant une famille dénombrable de feuilletages avec même partie homogène de degré 3 qui ne sont pas équivalents.

Terminons ce paragraphe par le cas exceptionnel. On considère sur $\mathbb{P}_{\mathbb{C}}^{3}$ le feuilletage $\mathcal{F}_{\Gamma}$ dont les feuilles sont les niveaux de la fonction rationnelle $\frac{F^{2}}{G^{3}}$ où $F$ et $G$ sont donnés en coordonnées homogènes par : $F=x_{3} x_{4}^{2}-x_{1} x_{2} x_{4}+\frac{x_{1}^{3}}{3}, G=x_{2} x_{4}-\frac{x_{1}^{2}}{2}$. Il s'agit d'un feuilletage de degré 2 , donné par $\Omega_{3}=\frac{1}{x_{4}}(2 G d F-3 F d G)$, appelé feuilletage exceptionnel; il est exceptionnel à plusieurs titres. L'orbite de $\mathcal{F}_{\Gamma}$ sous l'action du groupe d'automorphismes $\operatorname{Aut}\left(\mathbb{P}_{\mathbb{C}}^{3}\right)$, ou plutôt sa fermeture, est une composante irréductible de l'espace des feuilletages de degré 2 sur $\mathbb{P}_{\mathbb{C}}^{3}[9]$. D'autre part ses feuilles sont des surfaces de degré 6 , ce qui en un certain sens est un degré anormal pour un feuilletage de degré 2 . Son lieu singulier $\Gamma$ est constitué d'une droite, d'une conique et d'une cubique gauche tangentes en un point $q$ [9]. Soit $H \simeq \mathbb{P}_{\mathbb{C}}^{2}$ un hyperplan générique, i.e. transverse à $\Gamma$. La restriction de $\mathcal{F}_{\Gamma}$ à $H$ est un feuilletage de degré 2, qui a donc sept points singuliers comptés avec multiplicité. Comme $\Gamma-\{q\}$ est constitué des lignes de Kupka et $H$ est transverse à $\Gamma$, les six points de $H \cap \Gamma$ sont des points singuliers de $\left.\mathcal{F}_{\Gamma}\right|_{H}$ qui ont pour nombre de Milnor 1 . Il y a donc un autre point $p \in H$ singulier pour $\left.\mathcal{F}_{\Gamma}\right|_{H}$ avec nombre de Milnor égal a 1. Comme $p$ n'appartient pas à $\Gamma$, le feuilletage $\mathcal{F}_{\Gamma}$ a une intégrale première local $\varphi$ submersive en $p$, et la restriction $\left.\varphi\right|_{H}$ est nécéssairement de Morse en $p$. Ainsi $p$ est un centre de $\left.\mathcal{F}_{\Gamma}\right|_{H}$. Supposons $H$ donné par $x_{4}=a x_{1}+b x_{2}+c x_{3}=L$, avec $a b c \neq 0$, et notons $f$ et $g$ les restrictions de $F$ et $G$ à $H: f=F\left(x_{1}, x_{2}, x_{3}, L\right), g=G\left(x_{1}, x_{2}, x_{3}, L\right)$. Le feuilletage $\left.\mathcal{F}_{\Gamma}\right|_{H}$ est défini par la forme :

$$
\omega_{3}^{H}=\omega_{3}=\frac{1}{L}(2 g d f-3 f d g) \in \Omega^{1}\left(\mathbb{C}^{3}, 0\right) .
$$

Le feuilletage $\mathcal{F}_{\omega_{3}}$ a, pour $L$ générique, sept droites singulières dont six sont de type Kupka et la septième de type centre, en dehors de 0. 
On considère maintenant la perturbation $\omega=\omega_{3}+\cdots$, de 3 -jet $\omega_{3}$, défini par :

$$
\omega=\omega_{3}+2 f g d L=\frac{1}{L}\left(2 g d f-3 f d g+f g d L^{2}\right) .
$$

Cette 1 -forme est intégrable puisque :

$$
\omega=\frac{f g}{L}\left(2 \frac{d f}{f}-3 \frac{d g}{g}+d L^{2}\right) .
$$

Notons que pour $H$ générique, le point $p \in H$ est en dehors des zéros de $F$ et $G$ et donc de $\left[f^{-1}(0)\right]$ et $\left[g^{-1}(0)\right]$. Si l'on procède à l'éclatement de $\omega$ par $E=\left(y_{1}, y_{1} y_{2}, y_{1} y_{3}\right)$ on obtient :

$$
E^{*} \omega=\frac{y_{1}^{5} \tilde{f} \tilde{g}}{y_{1}\left(a+b y_{2}+c y_{3}\right)}\left(2 \frac{d \tilde{f}}{\tilde{f}}-3 \frac{d \tilde{g}}{\tilde{g}}+d y_{1}^{2}\left(a+b y_{2}+c y_{3}\right)^{2}\right)
$$

avec $f \circ E=y_{1}^{3} \tilde{f}, g \circ E=y_{1}^{2} \tilde{g}$. On note que $\tilde{f}$ et $\tilde{g}$ sont des unités en $p$ et comme $\left.\mathcal{F}_{\Gamma}\right|_{H}$ a un centre en $p$, le 1-jet de $2 \frac{d \tilde{f}}{\tilde{f}}-3 \frac{d \tilde{g}}{\tilde{g}}$ en $p$ est de type $u d v+v d u$ pour un bon choix de coordonnées $u, v$. Par suite le feuilletage $E^{-1} \mathcal{F}_{\omega}$ a en $p$ une singularité isolée; en résulte que l'ensemble singulier de $\mathcal{F}_{\omega}$ est constitué de six courbes lisses et non sept comme $\mathcal{F}_{\omega_{3}}$. Par suite $\mathcal{F}_{\omega}$ et $\mathcal{F}_{\omega_{3}}$ ne sont pas conjugués.

On peut aussi considérer les feuilletages $\mathcal{F}_{\omega^{k}}$ associés aux 1 -formes :

$$
\omega^{k}=\omega_{3}+f g k L^{k-1} d L, \quad k \in \mathbb{N} .
$$

En étudiant la singularité $E^{-1} \mathcal{F}_{\omega^{k}, p}$ on constate que les feuilletages $\mathcal{F}_{\omega^{k}}$ ne sont pas holomorphiquement conjugués. Ceci indique en particulier que le feuilletage $\mathcal{F}_{\omega_{3}}$ n'est pas de détermination finie; il est probable par contre que les $\mathcal{F}_{\omega^{k}}$ pour $k \geq 1$ le soient.

\section{Conclusion}

Rappelons que la classification des feuilletages de degré deux de $\mathbb{P}_{\mathbb{C}}^{2}$ n'ayant qu'une singularité (donc à nombre de Milnor 7) est faite dans [8]; tous sont définis par une 1-forme fermée méromorphe sauf un modèle ayant une singularité noeud-col, donc satisfaisant la condition de Kupka. Tous les résultats qui précèdent sont résumées dans le Théorème $\mathrm{A}$ présenté dans l'introduction. On obtient en particulier la classification des feuilletages $\mathcal{F}_{\omega}$ sur $\mathbb{C}^{3}, 0$ à partie initiale $\operatorname{In}(\omega)$ dicritique de degré 3 , Cod Sing $\operatorname{In}(\omega) \geq 2$ (modulo la condition de non résonnance locale $b \notin \mathbb{Q}$ ).

Voici maintenant une liste de problèmes ouverts.

1- Traiter le cas résonnant d'une singularité de type $\Omega_{2}$ avec $b \in \mathbb{Q}$.

2 - Soit $\omega_{\nu}$ une 1 -forme intégrable homogène de degré $\nu$, Cod Sing $\omega_{\nu} \geq 2$; on considère l'ensemble

$$
\mathcal{F} \operatorname{IN}\left(\omega_{\nu}\right):=\left\{\text { feuilletages } \mathcal{F}=\mathcal{F}_{\omega} \text { avec } \operatorname{In}(\omega)=\omega_{\nu}\right\} .
$$

Sur $\mathcal{F} \operatorname{IN}\left(\omega_{\nu}\right)$, il y a la relation d'équivalence de conjugaison holomorphe : $\mathcal{F}_{\omega} \sim \mathcal{F}_{\omega^{\prime}}$ si et seulement si il existe un difféomorphisme $\phi$ tangent à l'identité tel que $\phi^{*} \mathcal{F}_{\omega}=\mathcal{F}_{\omega^{\prime}}$. Décrire l'espace de module $\mathcal{M}\left(\omega_{\nu}\right)=\mathcal{F} \operatorname{IN}\left(\omega_{\nu}\right) / \sim$; par exemple si $\omega_{\nu}$ satisfait le point 3 du Théorème $\mathrm{A}$ alors $\mathcal{M}\left(\omega_{\nu}\right)$ se réduit à un point. Par contre nous venons de voir des exemples où $\mathcal{M}\left(\omega_{\nu}\right)$ est infini $\left(\omega_{\nu}=Q_{1} d Q_{2}-Q_{2} d Q_{1}\right.$ comme ci-dessus).

3- Généraliser les énoncés du Théorème A aux dimensions supérieures à 3. La classification de 9] permet de se ramener au cas où la partie initiale ne dépend que de 3 variables.

4- Donner une démonstration géométrique, i.e. non calculatoire, du Théorème de Dulac. 


\section{RÉFÉRENCES}

[1] Camacho, C.; Lins Neto, A. The topology of integrable differential forms near a singularity. Publ. Math. IHES, 55 no.1 (1982) 5-35.

[2] Camacho, C.; Lins Neto, A.; Sad, P. Topological invariants and equidesingularization for holomorphic vector fields. Journ. of Diff. Geo., 20 no.1 (1984) 143-174.

[3] Cano, F.; Cerveau, D.; Déserti, J. Théorie élémentaire des feuilletages holomorphes singuliers. Editions Belin, ISBN 978-2-7011-7484-6 (2013).

[4] Cano, F.; Corral, N. Absolutely Dicritical Foliations. Int. Math. Res. Not., 8 (2011) 1926-1934

[5] Cartan, H. Euvres. Vol. I, II, III. Springer-Verlag, Berlin (1979). Edité par Reinhold Remmert et Jean-Pierre Serre.

[6] Casale, G. Suites de Godbillon-Vey et intégrales premières. C. R. Acad. Sci. Paris Sér. I Math., 335 no.12 (2002) 1003-1006.

[7] Cerveau, D. Un théorème d'existence d'intégrales premières multiformes dans le cas dicritique. C. R. Acad. Sci. Paris Sér. I Math., 297, no.2 (1983) 105-107.

[8] Cerveau, D.; Déserti, J.; Garba Belko, D.; Meziani, R. Géométrie classique des feuilletages de degré deux. Bull. Braz. Math. Soc., 41, no.2 (2010) 161-198.

[9] Cerveau, D.; Lins Neto, A. Irreducible components of the space of holomorphic foliations of degree two in CP $(n), n \geq 3$. Annals of Math., 143 no.3 (1996) 577-612.

[10] Cerveau, D.; Lins Neto, A.; Loray, F.; Pereira, J.-V.; Touzet, F. Complex codimension one singular foliations and Godbillon-Vey sequences. Moscow Math. Journ. 7 no.1 (2007) 21-54.

[11] Cerveau, D.; Loray, F. Un théorème de Frobenius singulier via l'arithmétique élémentaire. J. Number Theory 68 no.2, (1998) 217-228.

[12] Cerveau, D.; Mattei, J.-F. Formes intégrables holomorphes singulières. Astérisque 97, Soc. Math. de France (1982)

[13] Cerveau, D.; Mozo-Fernandez, J. Classification analytique des feuilletages singuliers réduits de codimension 1 en dimension $n \geq 3$. Ergod. Theory Dynam. Syst., 22 no.4 (2002) 1041-1060.

[14] Dulac, H. Détermination et intégration d'une certaine classe d'équations différentielles ayant pour point singulier un centre. Bull. des Sc. Math. 32 (1908).

[15] Hironaka, H.; Matsumura, H. Formal functions and formal embeddings. J. Math. Soc. Japan, 20 no.1-2 (1968) $52-82$.

[16] Jouanolou, J. P. Équations de Pfaff Algébriques. Lect. Notes in Math., v. 708 (1979).

[17] Kupka, I. Singularities of integrable Pffafian forms. Proc. Nat. Acad. Sciences, 52 (1964).

[18] Lins Neto, A. Finite determinacy of germs of integrable 1-forms in dimension 3 (a special case). Lect. Notes In Math., v. 1007 (1980) 480-497.

[19] Loray, F. A Preparation Theorem for codimension one foliations. Annals of Math., 163 no.2 (2006) 709-722.

[20] Loray, F.; Pereira, J.-V.; Touzet, F. Representations of quasiprojective groups, flat connections and transversely projective foliations. hal-00942861 (2014).

[21] Malgrange, B. Frobenius avec singularité I : codimension 1. Publ. Math. IHES, 46 no.1 (1976) 163-173.

[22] Martinet, J.; Ramis, J.-P. Problèmes de modules pour des équations différentielles non linéaires du premier ordre. Publ. Math. IHES, 55 no.1 (1982) 63-164.

[23] Martinet, J.; Ramis, J.-P. Classification analytique des équations différentielles non linéaires résonnantes du premier ordre. Ann. Sci. de l'École Norm. Sup., 16 no.4 (1983) 571-621.

[24] Mattei, J.-F.; Moussu, R. Holonomie et intégrales premières. Ann. Sci. de l'École Norm. Sup., 13 no.4 (1980) 469-523.

[25] Saito, K. On a generalization of de Rham lemma. Ann. de l'Inst. Fourier, 26 no.2 (1976) 165-170.

[26] Singer, M. Liouvillian first integrals of differential equations. Trans. Amer. Math. Soc., 333 no.2 (1992) 673-688.

Dominique Cerveau, Institut Universitaire de France et IRMar, Université de Rennes 1, Campus de Bealieu, 35042 Rennes Cedex France.

E-mail address: dominique.cerveau@univ-rennes1.fr

Alcides Lins Neto, impa, Estrada Dona Castorina 110, Rio de Janeiro, Brasil.

E-mail address: alcides@impa.br

Marianna Ravara-Vago, irmar, Université de Rennes 1, Campus de Beaulieu, 35402 Rennes Cedex France.

E-mail address: ravaravago@gmail.com 